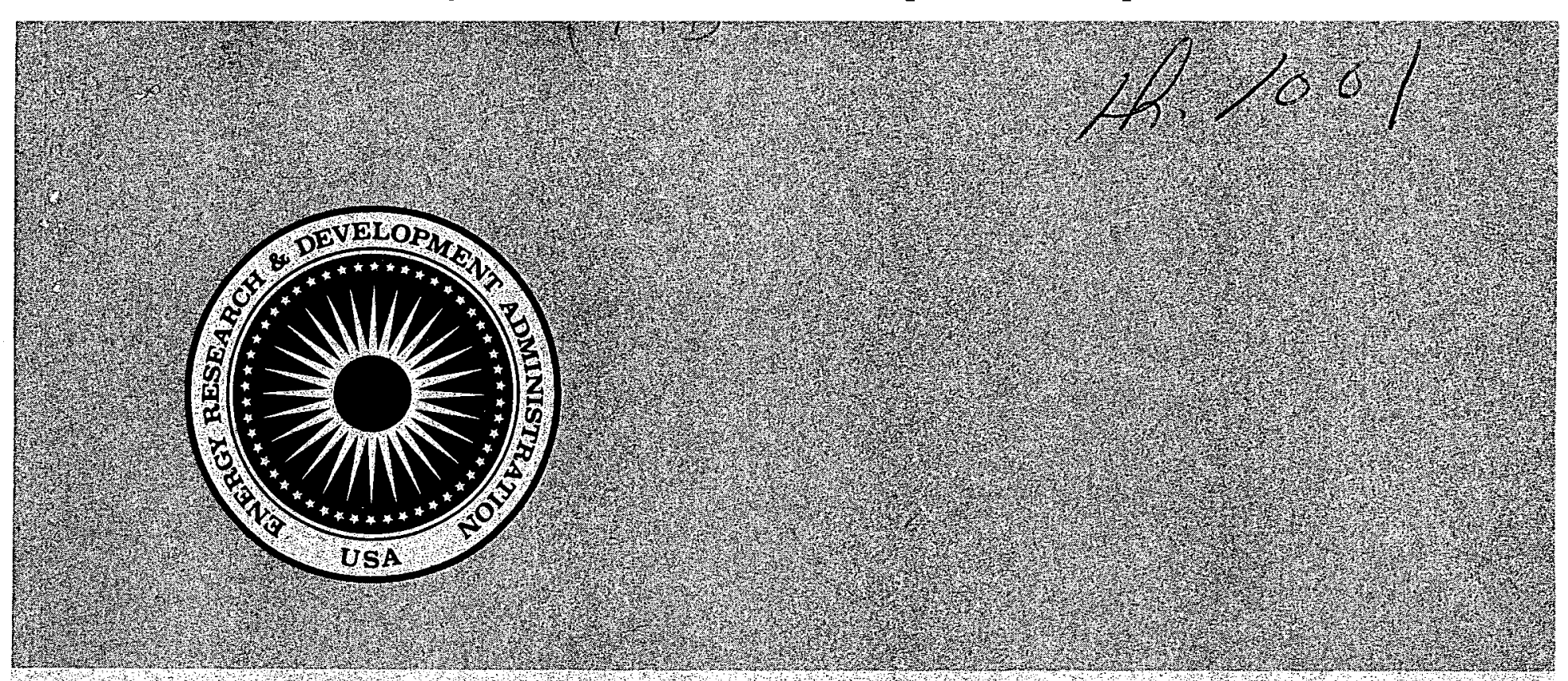

BERC/IC $77 / 1$

\title{
MASTER
}

\section{CARBON DIOXIDE SOURCES AND OILFIELDS IN A NINESTATE AREA OF THE SOUTHWEST UNITED STATES}

By

Robert E. Finney and Larman J. Heath

Date Published-April 1977

Bartlesville Energy Research Center

Energy Research and Development Administration

Bartlesville, Oklahoma 


\section{DISCLAIMER}

This report was prepared as an account of work sponsored by an agency of the United States Government. Neither the United States Government nor any agency Thereof, nor any of their employees, makes any warranty, express or implied, or assumes any legal liability or responsibility for the accuracy, completeness, or usefulness of any information, apparatus, product, or process disclosed, or represents that its use would not infringe privately owned rights. Reference herein to any specific commercial product, process, or service by trade name, trademark, manufacturer, or otherwise does not necessarily constitute or imply its endorsement, recommendation, or favoring by the United States Government or any agency thereof. The views and opinions of authors expressed herein do not necessarily state or reflect those of the United States Government or any agency thereof. 


\section{DISCLAIMER}

Portions of this document may be illegible in electronic image products. Images are produced from the best available original document. 


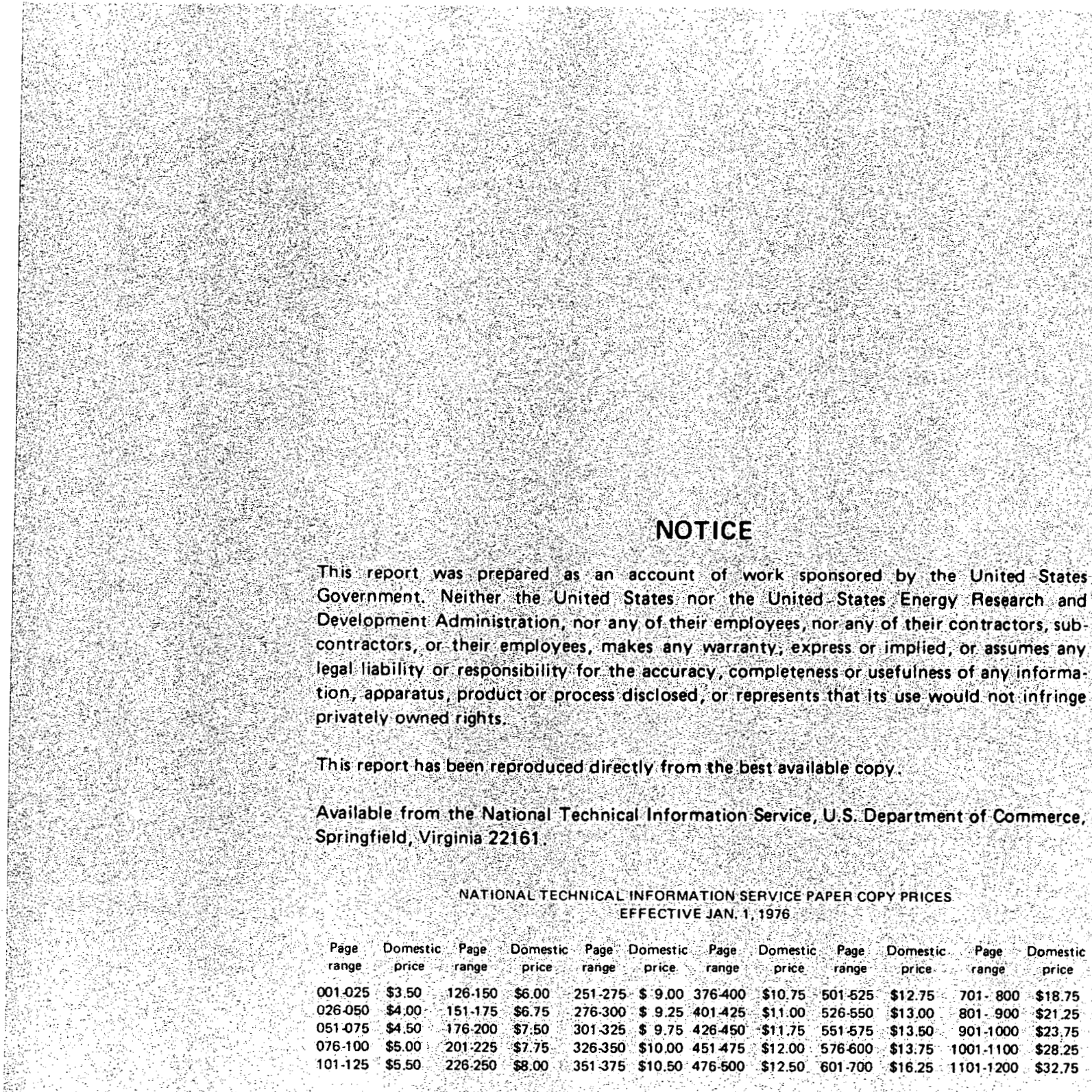

For additional pages add $\$ 4.50$ tor eact beginning 100 pages Add $\$ 250$ per copy for foreign price

Microfiche $\$ 3,00$ (domesticl $\$ 4$ so (foreign) 


\section{CARBON DIOXIDE SOURCES AND OILFIELDS \\ IN A NINE-STATE AREA OF THE SOUTHWEST UNITED STATES}

\section{By}

Robert E. Finney and Larman J. Heath

Bartlesville Energy Research Center

Bartlesville, Oklahoma

Date Published-April 1977

\section{UNITED STATES ENERGY RESEARCH \& DEVELOPMENT ADMINISTRATION} TECHNICAL INFORMATION CENTER 
Abstract ......................................... 1

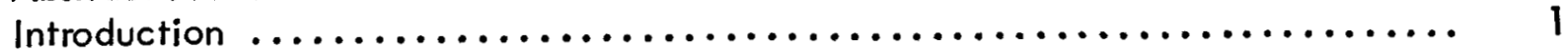

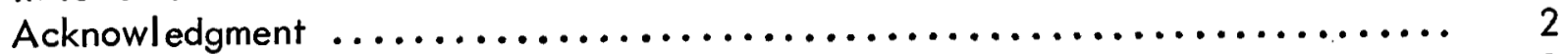

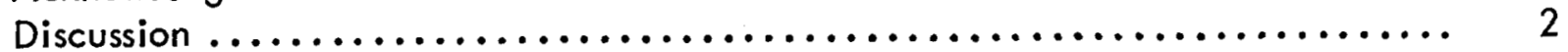

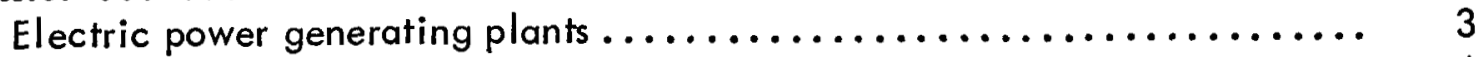

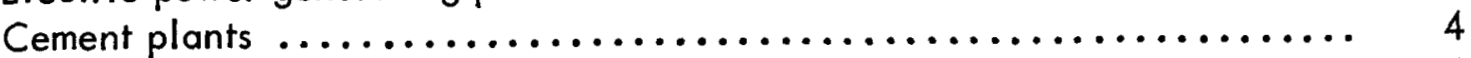

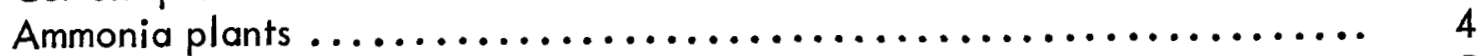

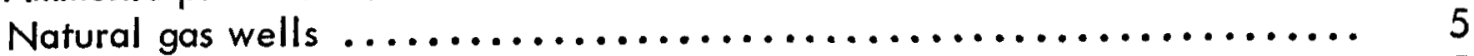

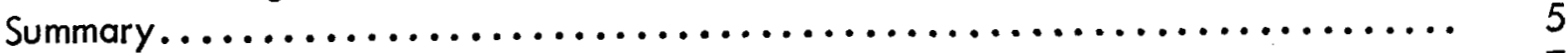

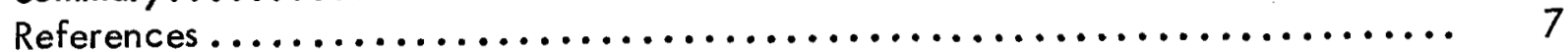

TABLES

No.

1. Carbon dioxide sources of supply in Arkansas .................... 9

2. Carbon dioxide sources of supply in Colorado .................. 10

3. Carbon dioxide sources of supply in Kansas ................... 12

4. Carbon dioxide sources of supply in Louisiana.................. 14

5. Carbon dioxide sources of supply in Mississippi . . . . . . . . . . . . . . 16

6. Carbon dioxide sources of supply in Missouri .................. 17

7. Carbon dioxide sources of supply in New Mexico................. 19

8. Carbon dioxide sources of supply in Oklahoma .................. 21

9. Carbon dioxide sources of supply in Texas .................... 22

\section{ILLUSTRATIONS}

Fig.

1. Oilfields and carbon dioxide sources in Arkansas ................. 27

2. Oilfields and carbon dioxide sources in Colorado................. 28

3. Oilfields and carbon dioxide sources in Kansas..................... 29

4. Oilfields and carbon dioxide sources in Louisiana ................ 30

5. Oilfields and carbon dioxide sources in Mississippi ............... 31

6. Oilfields and carbon dioxide sources in Missouri ................. 32

7. Oilfields and carbon dioxide sources in New Mexico ............... 33

8. Oilfields and carbon dioxide sources in Oklahoma............... 34

9. Oilfields and carbon dioxide sources in Texas................... 35 


\title{
CARBON DIOXIDE SOURCES AND OILFIELDS \\ IN A NINE-STATE AREA OF THE SOUTHWEST UNITED STATES
}

By

\author{
Robert E. Finney ${ }^{1}$ and Larman J. Heath ${ }^{2}$
}

\begin{abstract}
Data on the prospective available $\mathrm{CO}_{2}$ in a nine-State area of the Southwest were collected from scattered sources and compiled to make the information readily available to operators who may be interested in using $\mathrm{CO}_{3}$ techniques for enhanced oilfield recoveries. Maps of the oilfields in the area studied are provided so that location of supply source can be related to possible point of use.
\end{abstract}

The best potential sources of $\mathrm{CO}_{z}$ are flue gas from electric generating plants, flue gas from cement plants, output from ammonia plants and natural gas reservoirs. The $\mathrm{CO}_{2}$ sources are listed by states and includes the operator's name, location name, county, and average available amounts of $\mathrm{CO}_{2}$.

\section{INTRODUCTION}

Interest in tertiary methods for recovering more oil from known oil accumulations has centered on steam floods, fire floods, detergents, solvents, miscible agents, and various types of gas injection. The $\mathrm{CO}_{z}$ process, a gas-injection technique, has proved to be attractive for enhanced recovery in terms of both effectiveness of recovery and economics when $\mathrm{CO}_{2}$ is available locally.

Interest exists today in $\mathrm{CO}_{z}$ injections, not only in reservoirs containing high gravity-low viscosity oils, but also in those containing the low gravity-high viscosity heavy oils. In some reservoirs, this technique may be applied where other methods of enhanced recovery are not technically or economically feasible. $\mathrm{CO}_{2}$ floods, even when technically feasible in certain reservoirs, have been severely constrained by the unavailability of sufficient or low-cost sources of carbon dioxide.

The literature contains much information on the technical aspects, laboratory methods, and field investigations of $\mathrm{CO}_{z}$ flooding for enhanced oil recovery; however, scarcely

1 Petroleum Engineer

2 Project Leader 
any information has been published on the availability, location, and economics of obtaining $\mathrm{CO}_{2}$ from the various sources. The purpose of this study was to locate, identify, and estimate the quantities in potential $\mathrm{CO}_{3}$ sources. This study was limited to the nine-State area of the Southwest--Arkansas, Colorado, Kansas, Louisiana, Mississippi, Missouri, New Mexico, Oklahoma, and Texas.

\section{ACKNOWLEDGMENT}

The authors wish to acknowledge the assistance and cooperation by employees of the following companies: Oklahoma Gas and Electric Co., Public Service Company of Oklahoma, OKC Refiners, AIRCO Industries, SACROC Unit, Lone Star Gas Co., Agrico Chemical Co., Carbonic Chemical, Inc., Farmland Industries, Martin-Marietta Cement Co., General Portland Cement Co. Also gratefully acknowledged are J. Sheldon, AIRCO Industries; and L. Bourdon, OKC Refineries for their discussion of output capacities and source locations.

\section{DISCUSSION}

The following potential sources of carbon dioxide were investigated:

1. Flue gas output from electric-power-generating plants

2. Flue gas output from cement plants

3. Output from ammonia or urea plants

4. Natural gas reservoirs in the Southwest

5. Fermentation industries

6. Phosphate manufacturing

7. Limestone calcination

8. Flue gas output from refinery operations

From the above list, the best potential sources $(1)^{1}$ of carbon dioxide apparently are flue gas from electric-power-generating plants, flue gas from cement plants, output from ammonia or urea plants, and natural gas reservoirs which contain $\mathrm{CO}_{2}$ in the covered nine-State area. The relative small amounts of carbon dioxide available from the fermentation industry, phosphate manufacturing, and limestone calcination operations would seem to eliminate them from consideration as potential sources. Oil refineries discharge large amounts of flue gas to the atmosphere, but the required investments for gathering systems at the widely diversified operations would make them unattractive for sources of supply.

This study was addressed to the first four primary candidates in the above list as sources of supply.

1 Underlined numbers in parentheses refer to items listed in the reference at the end of this report. 
(a) $\mathrm{CO}_{2}$ flow rates from stack flue gas ranged from 1 million to 300 million cu $\mathrm{ft} / \mathrm{day}$ from gas-fired units and up to 2 billion cu $\mathrm{ft} /$ day from coal-fired units. The total flue gas output will range 8 to 15 times higher than the $\mathrm{CO}_{z}$ production from each plant (12). The $\mathrm{CO}_{2}$ output from each generating unit was estimated by multiplying a constant conversion factor of 0.14 to the megawatt output capacity for the gas-fired units and a factor of 0.84 to the megawatt output capacity for the coal-fired units $(10,11)$. The coal conversion factor is approximately six times larger than the gas conversion factor because coal-fired units require nine to ten times the air intake as the gas-fired units (10). Conversion factors were determined by dividing the $\mathrm{CO}_{2}$ outputs by each megawatt output from the individual plants (10). An average factor was determined from several sources. These conversion factors were obtained from data supplied by various power system companies and verified by the Environmental Protection Agency report on point source emissions. No determination was attempted for the total contaminant output or the economics involved in separating the undesirable elements from the flue gas.

(b) The location and output amounts were determined as point sources; but for convenience and map readability, they were totaled into county listings as indicated on each state map in figures 1 through 9. The indicated county amounts include the gas-fired generators and coal-fired generator outputs. The separations for each point source are included in tables 1 through 9. The oilfields and $\mathrm{CO}_{2}$ sources for each state are listed in figures 1 through 9 for quick referral of the proximity for county source of supply to any field of interest.

(c) The recovery of $\mathrm{CO}_{2}$ from flue gases, compressing it for pipeline transmission, and transportation analysis has not been sufficiently studied, so that an overall economic evaluation is not available. The degree of purity required for a $\mathrm{CO}_{2}$ injection system has not been fully determined. This determination would be significant to the overall economics of a recovery program. Limited information on present sources places estimated costs of $\mathrm{CO}_{2}$ separated from flue gas between $\$ 0.80$ and $\$ 1.25 / \mathrm{mcf}(13)$. This estimate does not include pressurization and transportation charges; These additional significant charges would be influenced by the proximity of source to reservoir and the degree of miscibility required for each reservoir.

(d) The largest concentrations of $\mathrm{CO}_{2}$ from flue gas are located at the coal-fired, electric-power-generating plants. (9); however, coal-fired units are generally in areas where oil and gas suppliers are not readily available. Consequently, transportation costs for $\mathrm{CO}_{2}$ could prove this large source of supply to be unattractive for most potential amenable reservoirs. 


\section{Cement Plants}

(a) $\mathrm{CO}_{2}$ flow rates vary from 35 million to 123 million cu ft/day (22). Total flue gas output will range 8 to 15 times higher than the $\mathrm{CO}_{2}$ flow rate. The $\mathrm{CO}_{2}$ production rate in Mcfd from each plant unit was estimated by applying a constant conversion factor of 90 to the cement output capacity in tons/day for gas-fired units and a constant conversion factor of 178 for the coal-fired units (22). Conversion data were supplied by various cement plant operators and verified by the "point source output emissions" supplied by the Environmental Protection Agency (11). No attempt was made to determine the output contaminants or the economics of separation of the undesirable elements from the flue gas output.

(b) The point sources and available amounts are listed by each state on enclosure maps shown in figures 1 through 9 and tables 1 through 9. The various state oilfields are shown on each state map in figures 1 through 9 so that easy referral from source of supply to the field of interest may be made.

(c) The same criteria and limitations expressed in the discussion of electric-powergenerating plants would apply to the economic evaluation of obtaining $\mathrm{CO}_{2}$ from cement plant flue gas output. Best available estimates place the undelivered and unpressurized $\mathrm{CO}_{2}$ gas between $\$ 0.80$ and $\$ 1.25 \mathrm{mcf}(\underline{13})$.

(d) The largest concentrations of available flue gas $\mathrm{CO}_{2}$ from cement plants are those using coal as a primary fuel. Several of these plant sites are near fields that are potentially amenable to enhanced recovery operations and could become attractive candidates for sources of $\mathrm{CO}_{2}$.

\section{Ammonia Plants}

(a) $\mathrm{CO}_{2}$ production from ammonia and urea plants vary from 1 million to 14 million $\mathrm{cu} \mathrm{ft} /$ day. The present source of supply is listed on state maps in figures 1 through 9 with all county sources combined from the separate sources. The sources (1) are listed in tables 1 through 9. This potential source of $\mathrm{CO}_{z}$ supply is limited in the nine-State area since present supply and demand for $\mathrm{CO}_{2}$ in the foodstuff and other industries are fairly well balanced. This limited source of supply would be further strained by an increasing demand from the enhanced oil recovery industry.

(b) Pure $\mathrm{CO}_{2}$ is typically handled through commercial suppliers and is presently available for delivery at $\$ 2$ to $\$ 2.35 / \mathrm{mcf}$. These prices were determined from a survey of the commercial suppliers and the price schedule from AIRCO Industrial Gas Co. (1). 
4. Natural Gas Wells

(a) The carbon dioxide flow rates from natural gas reservoirs varies from small amounts to 87 million cu $\mathrm{ft} /$ day with purities up to 99.6 percent. These estimated volumes were calculated based on percentage volumes of $\mathrm{CO}_{2}$ compared with total gas flow previously reported in the publications (2-8, 16-21). Although some volumes were verified by production from industry $\overline{\text { reports }}$ and other publications, no real significance or correlation to present deliverability or total reserves should be attached to the data shown in tables 1 through 9. The listing of volumes only suggests possible areas of $\mathrm{CO}_{2}$ reserves. Recently, industry has shown much interest in determining the deliverability and reserves for several areas and has spent considerable time and effort to exploit possible $\mathrm{CO}_{2}$ reserves. At least two companies have made in-depth studies on transportation installations that might be required in some remote area sources. However, their findings on exploration, development, and the analysis on transportation requirements are confidential and not available for publication. Limited information does indicate that natural sources could be the most attractive source of supply for $\mathrm{CO}_{2}$.

(b) The sources available for natural $\mathrm{CO}_{z}$ gas were not defined sufficiently to indicate on the maps in figures 1 through 9.

(c) Cost of natural $\mathrm{CO}_{2}$ would be highly influenced by the degree of purity of produced gas, the degree of compressibility required for transporting, and the proximity of supply source to fields of interest. Some experience has been gained from $\mathrm{CO}_{2}$ enhanced recovery operations in West Texas which use heretofore-waste $\mathrm{CO}_{2}$ produced by the separation process at various gasoline plants. This experience factor, coupled with the knowledge gained from natural foodstuff carbon dioxide and the wealth of information on the economic analysis of natural gas production, place the estimated cost of produced carbon dioxide somewhat below the costs for natural gas. Best estimates available place the range from $\$ 0.25$ to $\$ 1.00 / \mathrm{mcf}$ delivered (13).

\section{SUMMARY}

The demand for carbon dioxide miscible flooding techniques has been severely constrained because of the general unavailability of source of supply. The pure carbon dioxide and industrial suppliers in the nine-State area of interest have present deliverability capabilities of 80 to 100 million cu $\mathrm{ft} / \mathrm{day}$ (1). Additional sources must be found if carbon dioxide miscible enhanced recovery fechniques are to be expanded. Natural gas reservoirs offer an attractive alternative and should have the deliverability capability of several billion cu ft/day. Several large reserves have been identified, but these are located at considerable distances from the most favorable oil reservoirs of interest. 
Carbon dioxide recovery from flue gas operation may prove to be a viable alternate if relative inexpensive methods for extraction, compression, and transportation can be developed. Carbon dioxide deliverability from flue gas conversion can range up to 125 billion cu ft/day for the area studied. Electric generating power plants represent the largest concentrated sources with cement plants being a good alternative for consideration. 


\section{REFERENCES}

1. AIRCO Industrial Gases. Industrial Gas Facilities Directory. November 25, 1975, $9 \mathrm{pp}$.

2. Anderson, C. C., and H. H. Hinson. Helium-Bearing Natural Gases of the United States. Analyses and Analytical Methods. BuMines Bull. 486, 1951, $141 \mathrm{pp}$.

3. Boone, W. J., Jr. Helium-Bearing Natural Gases of the United States. Analyses and Analytical Methods. Supplement to Bulletin 486. BuMines Bull. 576, 1958, 117 pp.

4. Cardwell, L. E., and L. F. Benton. Analyses of Natural Gases, 1968. BuMines IC $8443,1969,169$ pp.

5. ___ Analyses of Natural Gases, 1969. BuMines IC 8475, 1970, 134 pp.

6. __. Analyses of Natural Gases, 1970. BuMines IC 8518, 1971, 130 pp.

7. ___ Analyses of Natural Gases, 1971. BuMines IC 8554, 1972, 163 pp.

8. __. Analyses of Natural Gases, 1972. BuMines IC 8607, 1973, 104 pp.

9. Federal Energy Admin., Trends in Power Plant Capacity and Utilization. U.S. Standard Regional Area Listing of Generating Units by State. April 1976, $169 \mathrm{pp}$.

10. Federal Power Commission. Steam-Electric Plant Air and Water Quality Control Data. Summary Report, March 1975, 170 pp.

11. Finney, R. E. Private Communication. Environmental Protection Agency, Point Source Emission Reports. 1976. Available upon request from the authors, ERDA, Bartlesville, Okla.

12. Kirk-Othmer. Encyclopedia of Chemical Technology. McGraw-Hill Book Co., Inc., New York, N. Y., 2nd Ed., 1964, vol. 4, pp. 353-369.

13. Lewin and Associates, Inc. The Potential and Economics of Enhanced Oil Recovery. FEA Contract No. CO-03-50222-000. Final Report. April 1976.

14. Miller, R. D., and G. P. Norrell. Analyses of Natural Gases of the United States, 1961. BuMines IC 8221, 1964, 148 pp. 
15. - Analyses of Natural Gases of the United States, 1962. BuMines IC 8239, 1964, $120 \mathrm{pp}$.

16. . Analyses of Natural Gases of the United States, 1963. BuMines IC 8241, $1965,102 \mathrm{pp}$.

17. Moore, B. J., R. D. Miller, and R. D. Shrewsbury. Analyses of Natural Gases of the United States, 1964. BuMines IC 8302, 1966, 144 pp.

18. Moore, B. J., and R. D. Shrewsbury. Analyses of Natural Gases of the United States, 1965. BuMines IC 8316, 1966, 181 pp.

19. - Analyses of Natural Gases, 1966. BuMines IC 8356, 1967, 130 pp.

20. - Analyses of Natural Gases, 1967. BuMines IC 8395, 1968, 187 pp.

21. Munnerlyn, R. D., and R. D. Miller. Helium-Bearing Natural Gases of the United States: Analyses. Second Supplement to Bulletin 486. BuMines Bull. $617,1963,93 \mathrm{pp}$.

22. Portland Cement Assoc., Skokie, Illinois, Cement Plant Information Summary. December 31, 1975, 18 pp. 
TABLE 1. - Carbon dioxide sources of supply in Arkansas

\begin{tabular}{|c|c|c|c|c|}
\hline Company & Unit & County & $\begin{array}{c}\text { Capacity, } \\
\text { MW }\end{array}$ & $\begin{array}{c}\mathrm{CO}_{2} \text { Output, } \\
M c f d\end{array}$ \\
\hline \multicolumn{5}{|c|}{$\mathrm{CO}_{2}$ OUTPUT FROM ELECTRIC GENERATING PLANTS } \\
\hline $\begin{array}{l}\text { Arkansas Electric Coop } \\
\text { Do. } \\
\text { Do. } \\
\text { Do. } \\
\text { Do. } \\
\text { Do. } \\
\text { Mid Southern Arkansas } \\
\text { Power and Light } \\
\text { Do. } \\
\text { Do. } \\
\text { Do. } \\
\text { Do. } \\
\text { Do. }\end{array}$ & $\begin{array}{l}\text { Augusta } \\
\text { Bailey } \\
\text { Camden } \\
\text { Fitzhugh } \\
\text { McClellan } \\
\text { Ozark } \\
\\
\text { Cecil Lynch } \\
\text { Ham Moses } \\
\text { Harvey Couch } \\
\text { L. Catherine } \\
\text { R. E. Ritchie } \\
\text { Blytheville }\end{array}$ & $\begin{array}{l}\begin{array}{c}\text { Woodruff } \\
\text { do. } \\
\text { do. }\end{array} \\
\text { Franklin } \\
\text { Ouachita } \\
\quad \text { do. } \\
\text { Pulaski } \\
\text { St. Francis } \\
\text { Lafayette } \\
\text { Hot Springs } \\
\text { Phillips } \\
\text { Mississippi }\end{array}$ & $\begin{array}{r}100 \\
122 \\
125 \\
59 \\
134 \\
50 \\
\\
217 \\
126 \\
140 \\
666 \\
845 \\
189\end{array}$ & $\begin{array}{r}14,000 \\
17,000 \\
17,500 \\
8,300 \\
18,800 \\
7,000 \\
\\
30,300 \\
17,500 \\
19,600 \\
93,000 \\
118,000 \\
26,000\end{array}$ \\
\hline \multicolumn{5}{|c|}{$\mathrm{CO}_{2}$ OUTPUT FROM CEMENT PLANTS } \\
\hline $\begin{array}{l}\text { Arkansas Cement } \\
\text { Ideal Cement }\end{array}$ & $\begin{array}{l}\text { Foreman } \\
\text { Okay }\end{array}$ & $\begin{array}{l}\text { Bevier } \\
\text { Pulaski }\end{array}$ & & $\begin{array}{l}86,000 \\
36,000\end{array}$ \\
\hline \multicolumn{5}{|c|}{$\mathrm{CO}_{z}$ PRODUCTION FROM CHEMICAL PLANTS } \\
\hline $\begin{array}{l}\text { Monsanto Chemical } \\
\text { Ark Chemical }\end{array}$ & $\begin{array}{l}\text { Blytheville } \\
\text { Helana }\end{array}$ & $\begin{array}{l}\text { Mississippi } \\
\text { Phillips }\end{array}$ & & $\begin{array}{r}200 \\
3,480\end{array}$ \\
\hline \multicolumn{5}{|c|}{$\mathrm{CO}_{2}$ OUTPUT FROM NATURAL GAS WELLS } \\
\hline $\begin{array}{l}\text { Carter Oil Co. } \\
\text { McAlester Fuel Co. } \\
\text { Barnsdall } \\
\text { Carter Oil Co. }\end{array}$ & $\begin{array}{l}\text { Big Creek } \\
\text { Dorcheat } \\
\text { Mc Kamie } \\
\text { Fauke }\end{array}$ & $\begin{array}{l}\text { Columbia } \\
\text { do. } \\
\text { Lafayette } \\
\text { Miller }\end{array}$ & & $\begin{array}{c}\text { NA } \\
93 \\
\text { NA } \\
180\end{array}$ \\
\hline
\end{tabular}

NA - Not available. 
TABLE 2. - Carbon dioxide sources of supply in Colorado

\begin{tabular}{|c|c|c|c|c|}
\hline Company & Unit & County & $\begin{array}{l}\text { Capacity, } \\
\text { MW }\end{array}$ & $\begin{array}{l}\mathrm{CO}_{2} \text { Output, } \\
\text { Mcfd }\end{array}$ \\
\hline \multicolumn{5}{|c|}{$\mathrm{CO}_{3}$ OUTPUT FROM ELECTRIC GENERATING PLANTS } \\
\hline $\begin{array}{l}\text { Central Telephone \& } \\
\text { Utility Co. } \\
\text { Do. } \\
\text { City of Colorado } \\
\text { Springs } \\
\text { Do. } \\
\text { Colo.-Ute Electric } \\
\text { Association } \\
\text { Do. } \\
\text { City of Lamar } \\
\text { Public Service Co. of } \\
\text { Colorado } \\
\text { Do. } \\
\text { Do. } \\
\text { Do. } \\
\text { Do. } \\
\text { Do. } \\
\text { Do. } \\
\text { Do. } \\
\text { Tri-State Generating } \\
\text { \& Transmission } \\
\text { Western Colorado } \\
\text { Power Co. }\end{array}$ & $\begin{array}{l}\text { Pueblo New } \\
\text { W. N. Clark } \\
\text { Drake } \\
\text { G. Birdsal } \\
\text { Bullock } \\
\text { Nucla } \\
\text { Lamar } \\
\text { Alamosa } \\
\text { Arapohoe } \\
\text { Cameo } \\
\text { Cherokee } \\
\text { Comanche } \\
\text { Valmont } \\
\text { do. } \\
\text { Zuni } \\
\text { Wray } \\
\text { Bullock }\end{array}$ & $\begin{array}{c}\begin{array}{c}\text { Fremont } \\
\text { do. }\end{array} \\
\text { El Paso } \\
\text { do. } \\
\text { Montrose } \\
\text { do. } \\
\text { Prowers } \\
\text { Alamosa } \\
\text { Denver } \\
\text { Mesa } \\
\text { Adams } \\
\text { Pueblo } \\
\text { Boulder } \\
\text { do. } \\
\text { Denver } \\
\text { do. } \\
\text { Montrose }\end{array}$ & $\begin{array}{c}34 \\
41 \\
\\
286^{c} \\
56 \\
12 \\
57 \\
35 \\
\\
38 \\
240^{c} \\
75^{c} \\
733^{c} \\
700^{c} \\
180^{c} \\
166 \\
108 \\
\\
202 \\
13\end{array}$ & $\begin{array}{r}5,200 \\
34,000 \\
\\
240,000 \\
7,800 \\
10,000 \\
48,000 \\
4,900 \\
\\
5,300 \\
202,000 \\
63,000 \\
613,000 \\
566,000 \\
151,000 \\
23,000 \\
15,000 \\
\\
28,000 \\
1,800\end{array}$ \\
\hline \multicolumn{5}{|c|}{$\mathrm{CO}_{2}$ OUTPUT FROM CEMENT PLANTS } \\
\hline Martin Marietta & Lyons & Arapahoe & & 77,000 \\
\hline \multicolumn{5}{|c|}{$\mathrm{CO}_{2}$ PRODUCTION FROM CHEMICAL PLANTS } \\
\hline None & & & & \\
\hline \multicolumn{5}{|c|}{$\mathrm{CO}_{2}$ OUTPUT FROM NATURAL GAS WELLS } \\
\hline $\begin{array}{l}\text { U.S. Department of } \\
\text { Interior } \\
\text { Texaco }\end{array}$ & $\begin{array}{l}\text { Model Dome } \\
\text { White River - }\end{array}$ & $\begin{array}{l}\text { Las Animas } \\
\text { Rio Blanco }\end{array}$ & & $\begin{array}{l}600 \\
252\end{array}$ \\
\hline
\end{tabular}

See footnotes at end of table. 
TABLE 2. - Carbon dioxide sources of supply in Colorado--Continued

\begin{tabular}{l|l|l|r|r}
\hline \multicolumn{1}{c|}{ Company } & Unit & \multicolumn{1}{c|}{ County } & $\begin{array}{c}\text { Capacity, } \\
\text { MW }\end{array}$ & $\begin{array}{c}\mathrm{CO}_{2} \text { Output, } \\
\text { Mcfd }\end{array}$ \\
\hline \multicolumn{3}{c}{ COz } \\
\multicolumn{1}{c|}{ OUTPUT FROM NATURAL GAS WELLS--Cont'd. } \\
\hline Texaco & Tow Creek & Routt & & NA \\
Gulf Oil & & Montezuma & & 17,500 \\
Kerr McGee & Garmesa & Mesa & & NA \\
Amerado Petroleum & W. Salt Wash & do. & & NA \\
Superior & Douglas Creek & Rio Blanco & & NA \\
Lotus Oil Co. & Haxtun & Prowers & & 490 \\
Gulf Oil & South Canyon & Garfield & & 212 \\
$\quad$ Do. & & Jackson & & 13,200 \\
Frontier Refiners & White River & Rio Blanco & & 387 \\
Belco Petroleum & Lisbon S.E. & San Miguel & & NA \\
Pure Oil & do. & do. & & NA \\
\hline
\end{tabular}

c-coal plants

NA - Not available. 
TABLE 3. - Carbon dioxide sources of supply in Kansas

\begin{tabular}{l|l|l|l|l}
\hline Company & Unit & County & $\begin{array}{c}\text { Capacity, } \\
\mathrm{MW}\end{array}$ & $\begin{array}{c}\mathrm{CO}_{2} \text { Output, } \\
\text { Mcfd }\end{array}$ \\
\hline
\end{tabular}

$\mathrm{CO}_{2}$ OUTPUT FROM ELECTRIC GENERATING PLANTS

\begin{tabular}{|c|c|c|c|c|}
\hline $\begin{array}{l}\text { Central Telephone \& } \\
\text { Utility } \\
\text { Do. } \\
\text { Do. } \\
\text { Do. } \\
\text { Coffeyville Municipal } \\
\text { Light } \\
\text { Empire Electric Co. } \\
\text { City of Kansas City } \\
\text { Do. } \\
\text { Kansas City Power and } \\
\text { Light } \\
\text { Do. } \\
\text { Do. } \\
\text { Do. } \\
\text { Do. } \\
\text { Do. } \\
\text { Do. } \\
\text { Do. } \\
\text { Do. } \\
\text { McPherson Public Utility } \\
\text { Sunflower Electric Coop } \\
\text { City of Wellington } \\
\text { Wheatland Electric Coop } \\
\text { Winfield Light and Power }\end{array}$ & $\begin{array}{l}\text { Cimarron } \\
\text { River } \\
\text { Clifton } \\
\text { Judson Large } \\
\text { Mullergren } \\
\text { Coffeyville } \\
\text { Riverton } \\
\text { Kaw } \\
\text { Quinclore } \\
\text { La Cygne } \\
\text { Gordon Evans } \\
\text { Murry Gill } \\
\text { Neosha } \\
\text { Ripley } \\
\text { Abilene } \\
\text { Hutchinson } \\
\text { Lawrence } \\
\text { Tecumseh } \\
\text {-- } \\
\text { Garden City } \\
\text {--- } \\
\text { Garden City } \\
\text { Winfield }\end{array}$ & $\begin{array}{l}\text { Seward } \\
\text { Washington } \\
\text { Ford } \\
\text { Barton } \\
\text { Montgomery } \\
\text { Cherokee } \\
\text { Wyandotte } \\
\quad \text { do. } \\
\text { Linn } \\
\text { Sedgewick } \\
\quad \text { do. } \\
\text { Labette } \\
\text { Sedgewick } \\
\text { Dickinson } \\
\text { Reno } \\
\text { Douglas } \\
\text { Shawnee } \\
\text { McPherson } \\
\text { Finney } \\
\text { Sumner } \\
\text { Finney } \\
\text { Cowley }\end{array}$ & $\begin{array}{c}59 \\
78 \\
183 \\
133 \\
\\
73 \\
140 \\
144 \\
397 \\
\\
824^{c} \\
542 \\
370 \\
58 c \\
75 \\
111 \\
550 \\
661 \\
476 \\
105 \\
227 \\
57 \\
17 \\
56\end{array}$ & $\begin{array}{r}8,000 \\
11,000 \\
26,000 \\
19,000 \\
12,000 \\
20,000 \\
21,000 \\
51,000 \\
\\
692,000 \\
76,000 \\
52,000 \\
49,000 \\
7,000 \\
16,000 \\
77,000 \\
91,000 \\
63,000 \\
15,000 \\
32,000 \\
8,000 \\
2,400 \\
7,800\end{array}$ \\
\hline \multicolumn{5}{|c|}{$\mathrm{CO}_{2}$ OUTPUT FROM CEMENT PLANTS } \\
\hline $\begin{array}{l}\text { Ash Grove } \\
\text { General } \\
\text { Lone Star } \\
\text { Monarch } \\
\text { Universal Atlas }\end{array}$ & $\begin{array}{l}\text { Chanute } \\
\text { Fredonia } \\
\text { Bonner Springs } \\
\text { Humbolt } \\
\text { Independence }\end{array}$ & $\begin{array}{l}\text { Allen } \\
\text { Wilson } \\
\text { Leavenworth } \\
\text { Allen } \\
\text { Montgomery }\end{array}$ & & $\begin{array}{r}86,000 \\
35,000 \\
70,000 \\
103,000 \\
37,000\end{array}$ \\
\hline
\end{tabular}

See footnotes at end of table. 
TABLE 3. - Carbon dioxide sources of supply in Kansas--Continued

\begin{tabular}{|c|c|c|c|c|}
\hline Company & Unit & County & $\begin{array}{c}\text { Capacity, } \\
\text { MW }\end{array}$ & $\begin{array}{c}\mathrm{CO}_{2} \text { Output, } \\
\text { Mcfd }\end{array}$ \\
\hline \multicolumn{5}{|c|}{$\mathrm{CO}_{2}$ PRODUCTION FROM CHEMICAL PLANTS } \\
\hline $\begin{array}{l}\text { Arco } \\
\text { Farmland Industries } \\
\text { Midwest Solvent } \\
\text { F.M.C. }\end{array}$ & $\begin{array}{l}\text { Lawrence } \\
\text { Dodge City } \\
\text { Atchison } \\
\text { Lawrence }\end{array}$ & $\begin{array}{l}\text { Douglas } \\
\text { Ford } \\
\text { Atchison } \\
\text { Douglas }\end{array}$ & & $\begin{array}{l}3,400 \\
3,400 \\
2,600 \\
1,000\end{array}$ \\
\hline \multicolumn{5}{|c|}{$\mathrm{CO}_{2}$ OUTPUT FROM NATURAL GAS WELLS } \\
\hline Lee Phillips & & Rice & & NA \\
\hline
\end{tabular}

c-coal plants

NA - Not available. 
TABLE 4. - Carbon dioxide sources of supply in Louisiana

\begin{tabular}{l|l|l|l|l}
\hline Company & Unit & County & $\begin{array}{c}\text { Capacity, } \\
\text { MW }\end{array}$ & $\begin{array}{c}\mathrm{CO}_{2} \text { Output, } \\
\text { Mcfd }\end{array}$ \\
\hline
\end{tabular}

$\mathrm{CO}_{z}$ OUTPUT FROM ELECTRIC GENERATING PLANTS

\begin{tabular}{|c|c|c|c|c|}
\hline $\begin{array}{l}\text { Alexander Electric \& } \\
\text { Power } \\
\text { Cajun Electric Power } \\
\text { Coop } \\
\text { Do. } \\
\text { Southwestern Electric } \\
\text { Power } \\
\text { Do. } \\
\text { Central Louisiana } \\
\text { Electric Co. } \\
\text { Do. } \\
\text { Do. } \\
\text { Gulf States Utilities } \\
\text { Do. } \\
\text { Do. } \\
\text { Houma Light \& Water } \\
\text { Lafayette Utility System } \\
\text { Do. } \\
\text { Louisiana Power \& Light } \\
\text { Do. } \\
\text { Do. } \\
\text { Do. } \\
\text { New Orleans Public } \\
\text { Service } \\
\text { Do. } \\
\text { Do. } \\
\text { Commission } \\
\text { City of Morgan } \\
\text { City of Natchitoches } \\
\text { New Orleans Sewer } \\
\text { and Water } \\
\text { Opelousas Electric and } \\
\text { Water } \\
\text { Ruston Water and Light }\end{array}$ & 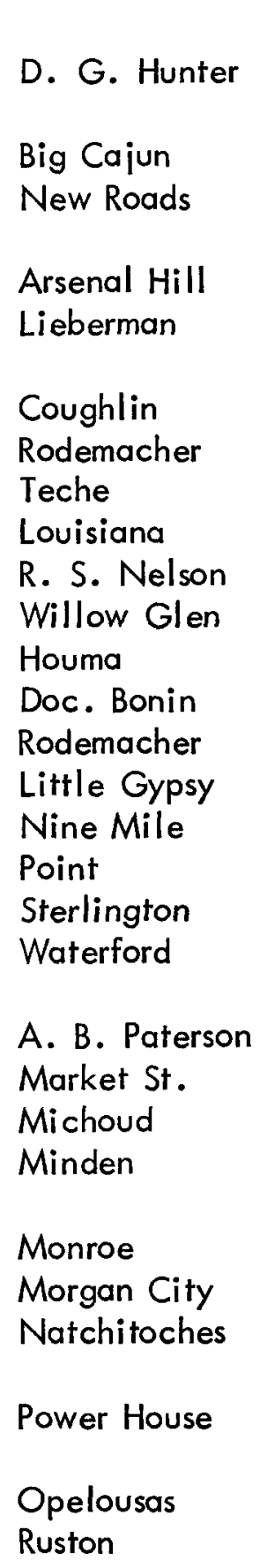 & $\begin{array}{l}\text { Rapides } \\
\begin{array}{c}\text { Pointe Coupee } \\
\text { do. }\end{array} \\
\text { Caddo } \\
\text { do. } \\
\text { Evangeline } \\
\text { do. } \\
\text { St. Mary } \\
\text { Baton Rouge } \\
\text { Calcasiew } \\
\text { Iberville } \\
\text { Terre Bonne } \\
\text { Lafayette } \\
\text { do. } \\
\text { St. Charles } \\
\text { Jefferson } \\
\text { Ouachita } \\
\text { St. Charles } \\
\text { Orleans } \\
\text { do. } \\
\text { do. } \\
\text { Webster } \\
\text { Ouachita } \\
\text { St. Mary } \\
\text { Natchitoches } \\
\text { Orleans } \\
\text { St. Landry } \\
\text { Lincoln }\end{array}$ & $\begin{array}{r}161 \\
276 \\
\\
477 \\
430 \\
426 \\
334 \\
899 \\
2,064 \\
94 \\
337 \\
51 \\
1,253 \\
1,827 \\
710 \\
576 \\
\\
226 \\
103 \\
905 \\
26 \\
\\
228 \\
67 \\
58 \\
41 \\
40 \\
187\end{array}$ & $\begin{array}{r}22,000 \\
38,000 \\
66,800 \\
60,000 \\
60,000 \\
46,800 \\
126,000 \\
289,000 \\
13,000 \\
47,000 \\
7,000 \\
175,000 \\
\\
256,000 \\
99,000 \\
80,600 \\
\\
33,000 \\
14,000 \\
127,000 \\
3,600 \\
\\
31,900 \\
9,400 \\
8,100 \\
5,700 \\
5,600 \\
26,000\end{array}$ \\
\hline
\end{tabular}


TABLE 4. - Carbon dioxide sources of supply in Louisiana--Continued

\begin{tabular}{|c|c|c|c|c|}
\hline Company & Unit & County & $\begin{array}{c}\text { Capacity, } \\
\text { MW }\end{array}$ & $\begin{array}{l}\mathrm{CO}_{2} \text { Output, } \\
\text { Mcfd } \\
\end{array}$ \\
\hline \multicolumn{5}{|c|}{$\mathrm{CO}_{2}$ OUTPUT FROM CEMENT PLANTS } \\
\hline $\begin{array}{l}\text { Lone Star } \\
\text { OKC }\end{array}$ & $\begin{array}{l}\text { New Orleans } \\
\text { do. }\end{array}$ & $\begin{array}{r}\text { Orlean } \\
\text { do. }\end{array}$ & & $\begin{array}{l}35,000 \\
50,000\end{array}$ \\
\hline \multicolumn{5}{|c|}{$\mathrm{CO}_{2}$ PRODUCTION FROM CHEMICAL PLANTS } \\
\hline $\begin{array}{l}\text { Allied Chemical } \\
\text { Agrico Chemical } \\
\text { Shell Oil Refinery } \\
\text { Monsanto Chemicals } \\
\text { American Cynamid } \\
\text { Monsanto Chemicals }\end{array}$ & $\begin{array}{l}\text { Gersmar } \\
\text { Donaldsville } \\
\text { Norco } \\
\text { Luling } \\
\text { Fortier } \\
\text { Donaldsville }\end{array}$ & $\begin{array}{l}\text { Ascension } \\
\text { Assumption } \\
\text { St. Charles } \\
\text { do. } \\
\text { do. } \\
\text { Assumption }\end{array}$ & & $\begin{array}{r}6,000 \\
10,000 \\
10,000 \\
14,000 \\
10,000 \\
10,000\end{array}$ \\
\hline \multicolumn{5}{|c|}{$\mathrm{CO}_{z}$ OUTPUT FROM NATURAL GAS WELLS } \\
\hline $\begin{array}{l}\text { Shell Oil Co. } \\
\text { Barnwell Drilling }\end{array}$ & $\begin{array}{l}\text { Vixen } \\
\text { do. }\end{array}$ & $\begin{array}{c}\text { Caldwell } \\
\text { do. }\end{array}$ & & $\begin{array}{l}408 \\
700\end{array}$ \\
\hline
\end{tabular}


TABLE 5. - Carbon dioxide sources of supply in Mississippi

\begin{tabular}{|c|c|c|c|c|}
\hline Company & Unit & County & $\begin{array}{c}\text { Capacity, } \\
\text { MW }\end{array}$ & $\begin{array}{c}\mathrm{CO}_{3} \text { Output, } \\
\text { Mcfd }\end{array}$ \\
\hline \multicolumn{5}{|c|}{$\mathrm{CO}_{2}$ OUTPUT FROM ELECTRIC GENERATING PLANTS } \\
\hline $\begin{array}{l}\text { Mississippi Power and } \\
\text { Light } \\
\text { Do. } \\
\text { Do. } \\
\text { Do. } \\
\text { Do. } \\
\text { Mississippi Power Co. } \\
\text { Do. } \\
\text { Mississippi Electric } \\
\text { Power Association } \\
\text { Do. }\end{array}$ & $\begin{array}{l}\text { Andrews } \\
\text { B. Wilson } \\
\text { Delta } \\
\text { Natchez } \\
\text { R. Brown } \\
\text { Sweatt } \\
\text { Watson } \\
\text { Moselle } \\
\text { Paulding }\end{array}$ & $\begin{array}{l}\text { Washington } \\
\text { Warren } \\
\text { Bolivar } \\
\text { Adams } \\
\text { Hinds } \\
\text { Lauderdale } \\
\text { Harrison } \\
\text { Jones } \\
\text { do. }\end{array}$ & $\begin{array}{r}750 \\
1,221 \\
208 \\
73 \\
390 \\
95 \\
696 \\
\\
227 \\
20\end{array}$ & $\begin{array}{r}105,000 \\
213,000 \\
29,000 \\
10,000 \\
54,000 \\
13,000 \\
97,000 \\
\\
32,000 \\
2,800\end{array}$ \\
\hline \multicolumn{5}{|c|}{$\mathrm{CO}_{z}$ OUTPUT FROM CEMENT PLANTS } \\
\hline $\begin{array}{l}\text { Marquette } \\
\text { Valley }\end{array}$ & $\begin{array}{l}\text { Branden } \\
\text { Redwood }\end{array}$ & $\begin{array}{l}\text { Rankin } \\
\text { Madison }\end{array}$ & & $\begin{array}{l}47,000 \\
59,000\end{array}$ \\
\hline \multicolumn{5}{|c|}{$\mathrm{CO}_{2}$ PRODUCTION FROM CHEMICAL PLANTS } \\
\hline Miscoa & Yazoo City & Madison & & 3,400 \\
\hline \multicolumn{5}{|c|}{$\mathrm{CO}_{2}$ OUTPUT FROM NATURAL GAS WELLS } \\
\hline $\begin{array}{l}\text { California Co. } \\
\text { CONOCO } \\
\text { Carter Oil } \\
\text { Skelly Oil } \\
\text { Chevron Oil } \\
\text { AMOCO }\end{array}$ & $\begin{array}{l}\text { Cranfield } \\
\text { Virlelia }\end{array}$ & $\begin{array}{l}\text { Adams } \\
\text { Madison } \\
\text { Rankin } \\
\text { do. } \\
\text { do. } \\
\text { Humphreys }\end{array}$ & & $\begin{array}{c}339 \\
2,800 \\
6,000 \\
\text { NA } \\
6,000 \\
\text { NA }\end{array}$ \\
\hline
\end{tabular}

NA - Not available. 
TABLE 6. - Carbon dioxide sources of supply in Missouri

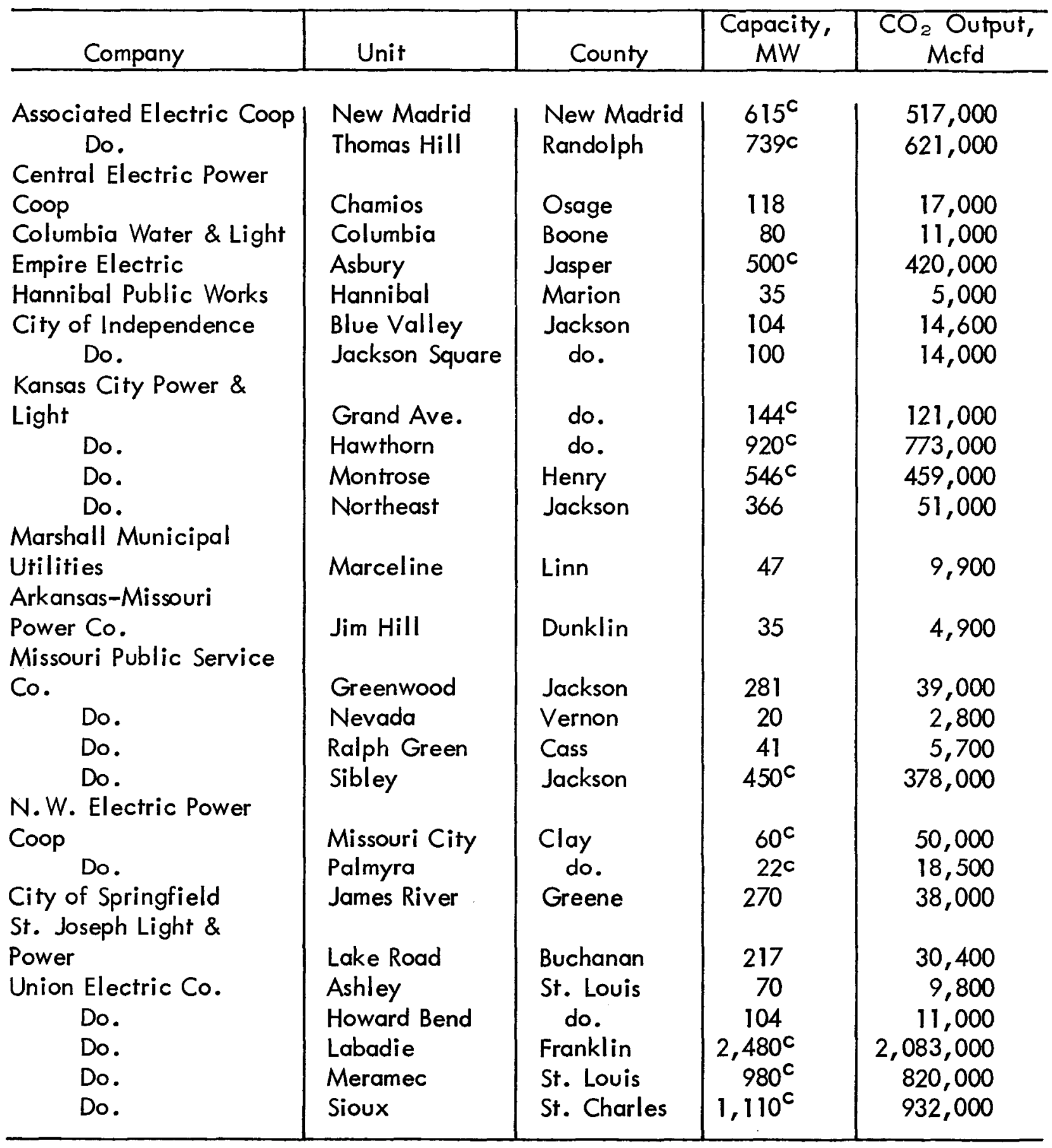

$\mathrm{CO}_{z}$ OUTPUT FROM CEMENT PLANTS

\begin{tabular}{l|l|l|l|r}
\hline $\begin{array}{l}\text { Alpha } \\
\text { Dundee }\end{array}$ & Clarksville & $\begin{array}{l}\text { St. Louis } \\
\text { Warren }\end{array}$ & & $\begin{array}{r}50,000 \\
216,000\end{array}$ \\
\hline
\end{tabular}

See footnotes at end of table. 
TABLE 6. - Carbon dioxide sources of supply in Missouri--Continued

\begin{tabular}{l|l|l|l|r}
\hline \multicolumn{1}{c|}{ Company } & \multicolumn{1}{c|}{ Unit } & \multicolumn{1}{c|}{ County } & $\begin{array}{c}\text { Capacity, } \\
\text { MW }\end{array}$ & $\begin{array}{c}\text { CO Output, } \\
\text { Mcfd }\end{array}$ \\
\hline \multicolumn{3}{c}{ CO2 OUTPUT FROM CEMENT PLANTS--Cont'd. } \\
\hline Lehigh & Union Bridge & St. Charles & & 81,000 \\
Marquette & Cape Girardeau & Cape Girardeau & & 52,000 \\
Missouri Portland & St. Louis & St. Louis & & 129,000 \\
Do. & Kansas City & Jackson & & 103,000 \\
River & Selma & Lincoln & & 203,000 \\
Universal Atlas & Hannibal & Clark & & 109,000 \\
\hline
\end{tabular}

c - coal plants 
TABLE 7. - Carbon dioxide sources of supply in New Mexico

\begin{tabular}{c|c|c|c|c}
\hline Company & Unit & County & $\begin{array}{c}\text { Capacity, } \\
M W\end{array}$ & $\begin{array}{c}\mathrm{CO}_{2} \text { Output, } \\
\text { Mcfd }\end{array}$ \\
\hline
\end{tabular}

$\mathrm{CO}_{z}$ OUTPUT FROM ELECTRIC GENERATING PLANTS

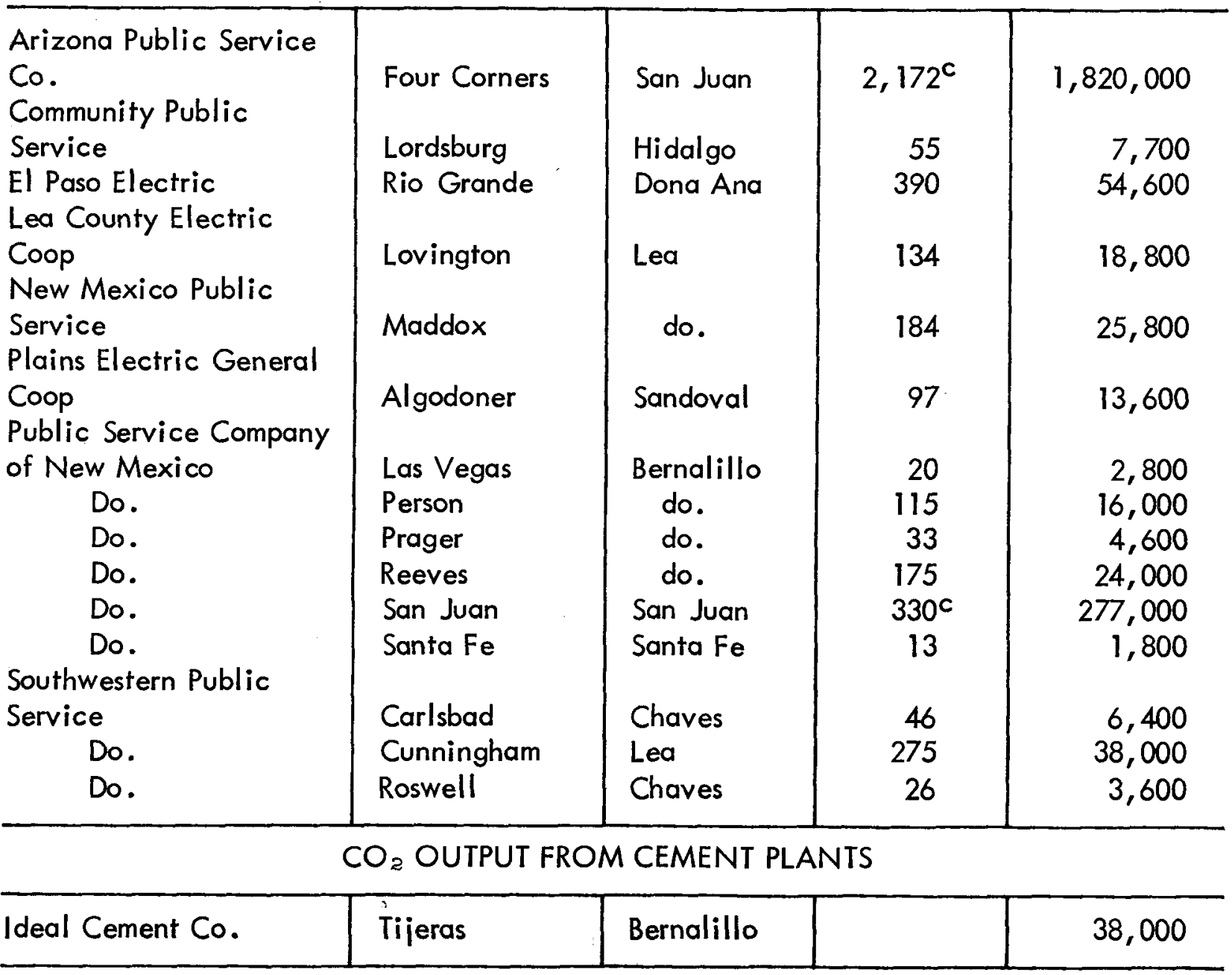

$\mathrm{CO}_{2}$ PRODUCTION FROM CHEMICAL PLANTS

\begin{tabular}{l|l|l|l|l}
\hline SEC & Solano & San Miquel & & 1,700 \\
\hline
\end{tabular}

$\mathrm{CO}_{3}$ OUTPUT FROM NATURAL GAS WELLS

\begin{tabular}{l|l|l|r|r}
\hline $\begin{array}{l}\text { Witt Ice \& Gas Co. } \\
\text { Arkansas Fuel \& Iron } \\
\text { NA }\end{array}$ & $\begin{array}{l}\text { Estancia } \\
\text { Wagon Mound } \\
\text { Maxwell }\end{array}$ & $\begin{array}{l}\text { Torrance } \\
\text { Mora } \\
\text { Colfax }\end{array}$ & & 2,350 \\
\hline
\end{tabular}

See footnotes at end of table. 
TABLE 7. - Carbon dioxide sources of supply in New Mexico--Continued

\begin{tabular}{|c|c|c|c|c|}
\hline Company & Unit & County & $\begin{array}{c}\text { Capacity, } \\
\text { MW }\end{array}$ & $\begin{array}{c}\mathrm{CO}_{2} \text { Oufput, } \\
\text { Mcfd }\end{array}$ \\
\hline \multicolumn{5}{|c|}{$\mathrm{CO}_{2}$ OUTPUT FROM NATURAL GAS WELLS--Continued } \\
\hline $\begin{array}{l}\text { Sierra Grande Oil } \\
\text { NA } \\
\text { American Producing Co. } \\
\text { Timmons Carbonic } \\
\text { Colo-Mer Gas Co. } \\
\text { Frontier } \\
\text { Core Oil } \\
\text { Robert Thorpe } \\
\text { Equity Oil } \\
\text { Belco } \\
\text { Delhi Taylor } \\
\text { Citgo } \\
\text { Tenneco }\end{array}$ & $\begin{array}{l}\text { Rogers No. } 1 \\
\text { Des Moines } \\
\text { Bueyeros } \\
\text { Derline No. } 1 \\
\text { Baca No. } 2 \\
\text { Fruita } \\
\text { San Miquel } \\
\text { Model Dome } \\
\text { Piceance Creek } \\
\text { S. E. Lisbon } \\
\text { McElmo } \\
\text { 3. Douglas Co. } \\
\text { McElmo }\end{array}$ & $\begin{array}{l}\text { Union } \\
\text { do. } \\
\text { Harding } \\
\text { do. } \\
\text { do. } \\
\text { San Juan } \\
\text { Mora } \\
\text { San Juan } \\
\text { do. } \\
\text { San Miquel } \\
\text { San Juan } \\
\text { do. } \\
\text { do. }\end{array}$ & & $\begin{array}{r}6,000 \\
N A \\
25,000 \\
3,000 \\
25,000 \\
N A \\
4 \\
N A \\
1,200 \\
6,600 \\
1,700 \\
5,760\end{array}$ \\
\hline
\end{tabular}

c-coal plants

NA - Not available. 
TABLE 8. - Carbon dioxide sources of supply in Oklahoma

\begin{tabular}{|c|c|c|c|c|}
\hline Company & Unit & County & $\begin{array}{c}\text { Capacity, } \\
\text { MW }\end{array}$ & $\begin{array}{l}\mathrm{CO}_{2} \text { Output, } \\
\text { Mcfd }\end{array}$ \\
\hline \multicolumn{5}{|c|}{$\mathrm{CO}_{2}$ OUTPUT FROM ELECTRIC GENERATING PLANTS } \\
\hline $\begin{array}{l}\text { Public Service Co. } \\
\text { Do. } \\
\text { Do. } \\
\text { Do. } \\
\text { Do. } \\
\text { Grand River Dam } \\
\text { Authority } \\
\text { Oklahoma Gas \& } \\
\text { Electric } \\
\text { Do. } \\
\text { Do. } \\
\text { Do. } \\
\text { Do. } \\
\text { Do. } \\
\text { Do. } \\
\text { Do. } \\
\text { Western Farmers Coop } \\
\text { Do. }\end{array}$ & $\begin{array}{l}\text { Comanche } \\
\text { Northeastern } \\
\text { Riverside } \\
\text { Tulsa } \\
\text { Weleetka } \\
\text { Chouteau } \\
\text { Arbuckle } \\
\text { Byng } \\
\text { Enid } \\
\text { Horseshoe Lake } \\
\text { Muskogee } \\
\text { Mustang } \\
\text { Osage } \\
\text { Seminole } \\
\text { Anadarko } \\
\text { Mooreland }\end{array}$ & $\begin{array}{l}\text { Comanche } \\
\text { Rogers } \\
\text { Tulsa } \\
\text { do. } \\
\text { Okfuskee } \\
\text { Mayes } \\
\text { Woods } \\
\text { Pontotoc } \\
\text { Garfield } \\
\text { Oklahoma } \\
\text { Muskogee } \\
\text { Canadian } \\
\text { Osage } \\
\text { Seminole } \\
\text { Caddo } \\
\text { Woodward }\end{array}$ & $\begin{array}{r}50 \\
75 \\
14 \\
56 \\
949 \\
208 \\
495 \\
42 \\
1,668 \\
152 \\
333\end{array}$ & $\begin{array}{r}16,800 \\
89,600 \\
126,000 \\
58,000 \\
10,900 \\
\\
7,000 \\
\\
10,500 \\
1,900 \\
7,800 \\
133,000 \\
30,000 \\
69,300 \\
5,900 \\
233,000 \\
21,000 \\
46,600\end{array}$ \\
\hline
\end{tabular}

$\mathrm{CO}_{2}$ OUTPUT FROM CEMENT PLANTS

\begin{tabular}{l|l|l|r|r}
\hline OKC & $\begin{array}{l}\text { Pryor } \\
\text { Ideal }\end{array}$ & $\begin{array}{l}\text { Rogers } \\
\text { Pontotoc } \\
\text { Martin Marietta }\end{array}$ & & 40,000 \\
Tulsa & 55,000 \\
& Tulsa & & 108,000 \\
\hline
\end{tabular}

$\mathrm{CO}_{z}$ PRODUCTION FROM CHEMICAL PLANTS

\begin{tabular}{l|l|l|l|c}
\hline Ni Pak, Inc. & Pryor & Rogers & & 2,100 \\
\hline \multicolumn{4}{c|}{ CO OUTPUT FROM NATURAL GAS WELLS } \\
\hline $\begin{array}{l}\text { Pure Oil Co. } \\
\text { Amarillo Natural Gas }\end{array}$ & $\begin{array}{l}\text { Keyes (3 wells) } \\
\text { Wildcat }\end{array}$ & $\begin{array}{l}\text { Cimarron } \\
\text { Texas }\end{array}$ & $\begin{array}{c}2,000+ \\
675\end{array}$ \\
\hline
\end{tabular}


TABLE 9. - Carbon dioxide sources of supply in Texas

\begin{tabular}{|c|c|c|c|c|}
\hline Company & Unit & County & $\begin{array}{c}\text { Capacity, } \\
\text { MW }\end{array}$ & $\begin{array}{c}\mathrm{CO}_{2} \text { Output, } \\
\text { Mcfd }\end{array}$ \\
\hline \multicolumn{5}{|c|}{$\mathrm{CO}_{2}$ OUTPUT FROM ELECTRIC GENERATING PLANTS } \\
\hline $\begin{array}{l}\text { Austin Electric } \\
\text { Department } \\
\text { Do. } \\
\text { Do. } \\
\text { Brazos Electric Coop } \\
\text { Do. } \\
\text { Do. } \\
\text { Do. } \\
\text { Do. } \\
\text { City of Bryan } \\
\text { Central Power \& Light } \\
\text { Do. } \\
\text { Do. } \\
\text { Do. } \\
\text { Do. } \\
\text { Do. } \\
\text { Do. } \\
\text { Do. } \\
\text { Southwestern Electric } \\
\text { Co. } \\
\text { Do. } \\
\text { Do. } \\
\text { Texas Utility Co. } \\
\text { Do. } \\
\text { Do. } \\
\text { Taylor Utility Co. } \\
\text { Do. } \\
\text { Do. } \\
\text { Do. } \\
\text { Do. } \\
\text { Denton Utility } \\
\text { El Paso Electric Co. } \\
\text { City of Garland } \\
\text { Go. } \\
\text { Do. }\end{array}$ & $\begin{array}{l}\text { Decker } \\
\text { Holby St. } \\
\text { Seaholm } \\
\text { Belton } \\
\text { No. Texas } \\
\text { Palo Pinto } \\
\text { R. W. Miller } \\
\text { Weatherford } \\
\text { Bryan } \\
\text { B. M. Davis } \\
\text { E. S. Joslin } \\
\text { J. S. Bates } \\
\text { La Palma } \\
\text { Laredo } \\
\text { L. C. Hill } \\
\text { Nueces Bay } \\
\text { Victoria } \\
\text { Knox Lee } \\
\text { Lone Star } \\
\text { Wilbur } \\
\text { Abilene } \\
\text { Concho } \\
\text { Fort Chamtom } \\
\text { Lake Pauline } \\
\text { Oak Creek } \\
\text { Paint Creek } \\
\text { Rio Pecos } \\
\text { San Angelo } \\
\text { Denton } \\
\text { Newman } \\
\text { C.E. Newman } \\
\text { R. Olinger } \\
\text { Lewis Creek } \\
\text { Neches } \\
\text { Sabrine }\end{array}$ & $\begin{array}{l}\text { Travis } \\
\text { do. } \\
\text { do. } \\
\text { Parker } \\
\text { do. } \\
\text { do. } \\
\text { Palo Pinto } \\
\text { do. } \\
\text { Brazos } \\
\text { Nueces } \\
\text { Calhoun } \\
\text { Hidalgo } \\
\text { Cameron } \\
\text { Webb } \\
\text { Nueces } \\
\text { do. } \\
\text { Victoria } \\
\text { Gregg } \\
\text { do. } \\
\text { Marion } \\
\text { Taylor } \\
\text { do. } \\
\text { do. } \\
\text { do. } \\
\text { do. } \\
\text { Haskell } \\
\text { Crockett } \\
\text { Tom Green } \\
\text { Denton } \\
\text { El Paso } \\
\text { Dallas } \\
\text { Collin } \\
\text { Montgomery } \\
\text { Jefferson } \\
\text { Orange } \\
\text { Oris. }\end{array}$ & $\begin{array}{r}725 \\
555 \\
120 \\
24 \\
76 \\
366 \\
395 \\
67 \\
241 \\
650 \\
240 \\
184 \\
260 \\
178 \\
543 \\
569 \\
520 \\
\\
550 \\
50 \\
879 \\
28 \\
51 \\
352 \\
47 \\
33 \\
235 \\
137 \\
137 \\
150 \\
470 \\
92 \\
204 \\
530 \\
425 \\
1450\end{array}$ & $\begin{array}{r}101,000 \\
77,700 \\
16,800 \\
3,400 \\
10,600 \\
52,000 \\
55,300 \\
9,400 \\
33,700 \\
94,000 \\
33,700 \\
25,800 \\
36,400 \\
24,900 \\
76,000 \\
79,700 \\
72,800 \\
77,600 \\
7,000 \\
123,000 \\
3,900 \\
7,000 \\
49,300 \\
6,600 \\
4,600 \\
32,900 \\
19,000 \\
19,000 \\
21,000 \\
65,800 \\
12,900 \\
28,600 \\
74,000 \\
59,500 \\
203,000\end{array}$ \\
\hline
\end{tabular}


TABLE 9. - Carbon dioxide sources of supply in Texas--Continued

\begin{tabular}{l|l|l|l|l}
\hline Company & Unit & County & $\begin{array}{c}\text { Capacity, } \\
\text { MW }\end{array}$ & $\begin{array}{c}\mathrm{CO}_{2} \text { Output, } \\
\text { Mcfd }\end{array}$ \\
\hline
\end{tabular}

$\mathrm{CO}_{z}$ OUTPUT FROM ELECTRIC GENERATING PLANTS--Cont'd.

Houston Light and Power Co.
Do.
Do.
Do.
Do.
Do.
Do.
Do.
Do.
Do.
Do.

Lower Colorado River Authority

Do.

Do.

Do.

Lubbock Power \& Light Do.

Medina Electric Coop

San Antonio Public Service

Do.

Do.

Do.

Do.

So. Texas Electric Coop Do.

Southwestern Public Service

Do.

Do.

Do.

Do.

Do.

Do.
Cedar Bayou

Champion

Deepwater

Gable Street

Greens Bayou

Hiram Clarke

P. H. Robinson

Sam Bertron

T. H. Wharton

W. A. Parish

Webster

Bastrop

Comal

S. Gideon

T. Ferguson

Holley Ave.

Plant No. 2

Peasall

Leon Creek

Mission Rd.

O. W. Somners

V. H. Braunig

W. B. Tuttle

San Rayburn

Victoria

Denver City

East Plant

Jones

Moore

Nichols

Plant $X$

Riverview

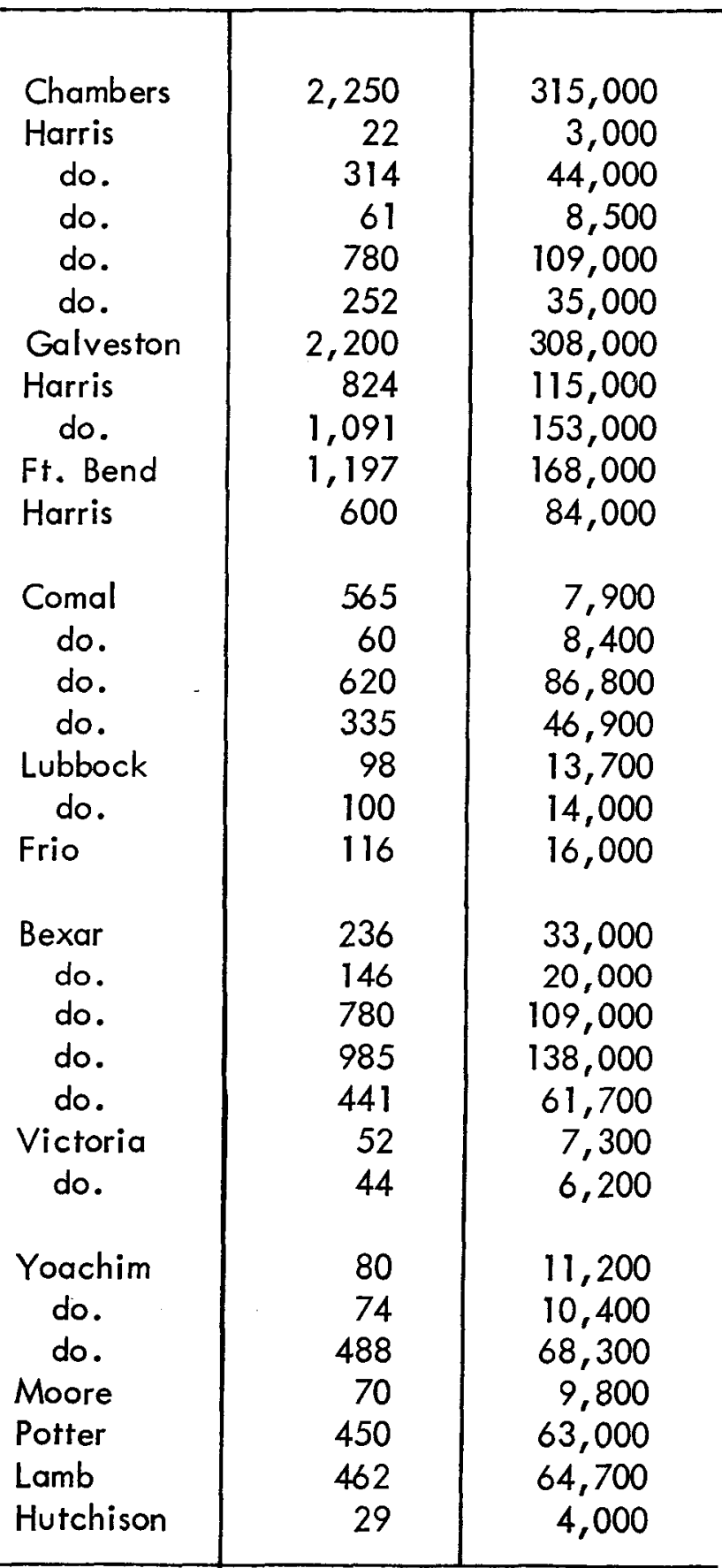


TABLE 9. - Carbon dioxide sources of supply in Texas--Continued

\begin{tabular}{c|c|c|c|c}
\hline Company & Unit & County & $\begin{array}{c}\text { Capacity, } \\
\mathrm{MW}\end{array}$ & $\begin{array}{c}\mathrm{CO}_{2} \text { Output, } \\
\text { Mcfd }\end{array}$ \\
\hline
\end{tabular}

$\mathrm{CO}_{z}$ OUTPUT FROM ELECTRIC GENERATING PLANTS--Cont'd.

\begin{tabular}{|c|c|c|c|c|}
\hline $\begin{array}{l}\text { Dallas Power \& Light } \\
\text { Do. } \\
\text { Do. } \\
\text { Do. } \\
\text { Do. } \\
\text { Texas Electric Service } \\
\text { Do. } \\
\text { Do. } \\
\text { Do. } \\
\text { Do. } \\
\text { Do. } \\
\text { Do. } \\
\text { Texas Power \& Light. } \\
\text { Do. } \\
\text { Do. } \\
\text { Do. } \\
\text { Do. } \\
\text { Do. } \\
\text { Do. } \\
\text { Do. } \\
\text { Do. } \\
\text { Do. }\end{array}$ & $\begin{array}{l}\text { Lake Hubbard } \\
\text { Mountain } \\
\text { Creek } \\
\text { North Lake } \\
\text { Parkdale } \\
\text { Eagle } \\
\text { Mountain } \\
\text { Handley } \\
\text { Graham } \\
\text { Morgan Creek } \\
\text { No. Main } \\
\text { Permian Basin } \\
\text { Wichita Falls } \\
\text { Big Brown } \\
\text { Collin } \\
\text { DeCordova } \\
\text { Lake Creek } \\
\text { Monticello } \\
\text { River Creek } \\
\text { Stryker Creek } \\
\text { Tradinghouse } \\
\text { Creek } \\
\text { Trinidad } \\
\text { Valley }\end{array}$ & $\begin{array}{l}\text { Dallas } \\
\text { do. } \\
\text { do. } \\
\text { do. } \\
\text { do. } \\
\text { Tarrant } \\
\text { do. } \\
\text { Young } \\
\text { Mitchell } \\
\text { Tarrant } \\
\text { Ward } \\
\text { Wichita } \\
\text { Freestone } \\
\text { Collin } \\
\text { Hood } \\
\text { McLennon } \\
\text { do. } \\
\text { Red River } \\
\text { Cherokee } \\
\text { McLennon } \\
\text { Henderson } \\
\text { Fannin }\end{array}$ & $\begin{array}{r}223 \\
890 \\
\\
1,028 \\
700 \\
327 \\
\\
665 \\
1,375 \\
610 \\
833 \\
121 \\
720 \\
26 \\
1,150 \\
153 \\
775 \\
317 \\
1,150 \\
110 \\
675 \\
\\
1,440 \\
325 \\
1,110\end{array}$ & $\begin{array}{r}31,000 \\
125,000 \\
144,000 \\
98,000 \\
45,800 \\
\\
93,000 \\
193,000 \\
85,000 \\
117,000 \\
16,900 \\
101,000 \\
3,600 \\
161,000 \\
21,400 \\
108,000 \\
44,000 \\
161,000 \\
15,000 \\
94,500 \\
202,000 \\
46,000 \\
150,000\end{array}$ \\
\hline \multicolumn{5}{|c|}{$\mathrm{CO}_{2}$ OUTPUT FROM CEMENT PLANTS } \\
\hline $\begin{array}{l}\text { Alpha } \\
\text { Capitol Aggregates } \\
\text { Centex } \\
\text { General } \\
\quad \text { Do. } \\
\text { Do. } \\
\text { Gifford Hill } \\
\text { Gulf Coast } \\
\text { Ideal }\end{array}$ & $\begin{array}{l}\text { Orange } \\
\text { San Antonio } \\
\text { Corpus Christi } \\
\text { Ft. Worth } \\
\text { Dallas } \\
\text { Houston } \\
\text { Midlothian } \\
\text { Houston } \\
\text { do. }\end{array}$ & $\begin{array}{l}\text { Orange } \\
\text { Bexar } \\
\text { Nueces } \\
\text { Tarrant } \\
\text { Dallas } \\
\text { Harris } \\
\text { Dallas } \\
\text { Harris } \\
\text { do. }\end{array}$ & & $\begin{array}{r}29,000 \\
64,000 \\
48,000 \\
123,000 \\
76,000 \\
94,000 \\
76,000 \\
45,000 \\
61,000\end{array}$ \\
\hline
\end{tabular}


TABLE 9. - Carbon dioxide sources of supply in Texas--Continued

\begin{tabular}{ll|l|l|c}
\hline Company & \multicolumn{1}{c|}{ Unit } & County & $\begin{array}{c}\text { Capacity, } \\
\text { MW }\end{array}$ & $\begin{array}{c}\text { COz Output, } \\
\text { Mcfd }\end{array}$ \\
\hline \multicolumn{3}{c|}{ COz } & OUTPUT FROM CEMENT PLANTS--Cont'd. \\
\hline Kaiser & San Antonio & Bexar & & \\
Lone Star & Houston & Harris & & 85,000 \\
Do. & Maryneal & do. & & 46,000 \\
San Antonio Port & Cementville & Bexar & & 46,000 \\
Southwestern & Odessa & Eaton & & 52,000 \\
Do. & Amarillo & Potter & & 22,000 \\
Texas Industries & Midlothian & Dallas & & 20,000 \\
Universal Atlas & Waco & McLennon & & 99,000 \\
& & & 39,000 \\
\hline
\end{tabular}

$\mathrm{CO}_{2}$ PRODUCTION FROM CHEMICAL PLANTS

\begin{tabular}{l|l|l|l|r}
\hline Tenneco Chemicals & Pasadena & Harris & & 4,250 \\
Southwest Cyrogenics & Deer Park & do. & & 1,800 \\
Diamond Shamrock & Dumas & Moore & & 1,000 \\
Southwest Cyrogenics & Victoria & Victoria & & 4,100 \\
\hline
\end{tabular}

$\mathrm{CO}_{2}$ OUTPUT FROM NATURAL GAS WELLS

\begin{tabular}{l|l|l|r}
\hline Humble Oil & Jourdanton & Otascosa & 35 \\
Texas Co. & Petrolia & Clay & 276 \\
Amerada & Seminole & Gaines & NA \\
ARCO & do. & do. & NA \\
California Co. & Glasscock & Glasscock & NA \\
Shell Oil & Yates & Pecos & NA \\
Canadian River Co. & Panhandle & Potter & NA \\
Group No. 1 Oil & Big Lake & Reagan & NA \\
Skelly Oil & Skelly-Grayson & do. & NA \\
Gulf Oil & McCamey & Upton & NA \\
Conoco & O'Brien & Winkler & NA \\
Tidewater & New Hope & Franklin & 1,100 \\
Sun Oil & John Creek & Hutchinson & 179 \\
Shell Oil & do. & Potter & NA \\
Mobil & Brown Bassett & Terrell & NA \\
\multicolumn{1}{c|}{ Do. } & L. M. Morrison & Bal Verde & NA \\
Sun Oil & & Crockett & do.700 \\
Texaco & & 2,000 \\
\hline
\end{tabular}

See footnote at end of table. 
TABLE 9. - Carbon dioxide sources of supply in Texas--Continued

\begin{tabular}{|c|c|c|c|c|}
\hline Company & Unit & County & $\begin{array}{c}\text { Capacity, } \\
M W\end{array}$ & $\begin{array}{c}\mathrm{CO}_{2} \text { Output, } \\
\mathrm{Mcfd}\end{array}$ \\
\hline \multicolumn{5}{|c|}{$\mathrm{CO}_{2}$ OUTPUT FROM NATURAL GAS WELLS--Cont'd. } \\
\hline $\begin{array}{l}\text { Stanolind Oil and Gas } \\
\text { Humble } \\
\text { Phillips Oil } \\
\text { Gulf Oil } \\
\text { Anton Oil } \\
\text { McElroy Ranch Co. } \\
\text { General American } \\
\text { Shell Oil Co. } \\
\text { Paul Teas } \\
\text { CONOCO Oil Co. } \\
\text { Mono Corp. } \\
\text { Sinclair } \\
\text { Shell \& Huber } \\
\text { Pan American } \\
\text { Tenneco } \\
\text { Industrial Gas } \\
\text { Forest Oil Co. } \\
\text { Texaco } \\
\text { Sunray DX } \\
\text { Gulf Oil } \\
\text { Union Oil } \\
\text { Couch Oil } \\
\text { J. L. Hamon } \\
\text { Humble Oil } \\
\text { Chevron } \\
\text { Superior Oil }\end{array}$ & $\begin{array}{l}\text { Stuart City } \\
\text { San Miquel } \\
\text { Puckett } \\
\text { Toyah } \\
\text { Winters } \\
\text { Wasson } \\
\text { Pawnee } \\
\text { Quinian } \\
\text { Brown Bassett } \\
\text { Wildcat } \\
\text { Brown Basseft } \\
\text { Rosita Wash } \\
\text { Miller } \\
\text { Cheapside } \\
\text { Yoakum } \\
\text { Linterna } \\
\text { Dunbar } \\
\text { Wolf Creek } \\
\text { Buffalo Wallow } \\
\text { do. } \\
\text { Wildcat } \\
\text { Box Church } \\
\text { Oates NE } \\
\text { Grey Ranch W } \\
\text { Mt. Vida }\end{array}$ & $\begin{array}{l}\text { La Salle } \\
\text { McMullen } \\
\text { Pecos } \\
\text { Reeves } \\
\text { Runnels } \\
\text { Stephans } \\
\text { Yoachim } \\
\text { Bee } \\
\text { Hunt } \\
\text { Terrell } \\
\text { Medina } \\
\text { Terrell } \\
\text { Duval } \\
\text { Crockett } \\
\text { Gonzales } \\
\text { DeWitt } \\
\text { Pecos } \\
\text { Rains } \\
\text { Pitkin } \\
\text { Hemphill } \\
\text { do. } \\
\text { Hopkins } \\
\text { Limestone } \\
\text { Pecos } \\
\text { do. } \\
\text { Reeves }\end{array}$ & & $\begin{array}{r}237 \\
90 \\
N A \\
4,000 \\
280 \\
70 \\
\text { NA } \\
502 \\
1,500 \\
15,300 \\
\text { NA } \\
4,990 \\
2,000 \\
1,350 \\
1,000 \\
260 \\
2,800 \\
2,200 \\
240 \\
34,000 \\
5,800 \\
8,500 \\
1,500 \\
3,400 \\
87,800 \\
35,000\end{array}$ \\
\hline
\end{tabular}

NA - Not available. 


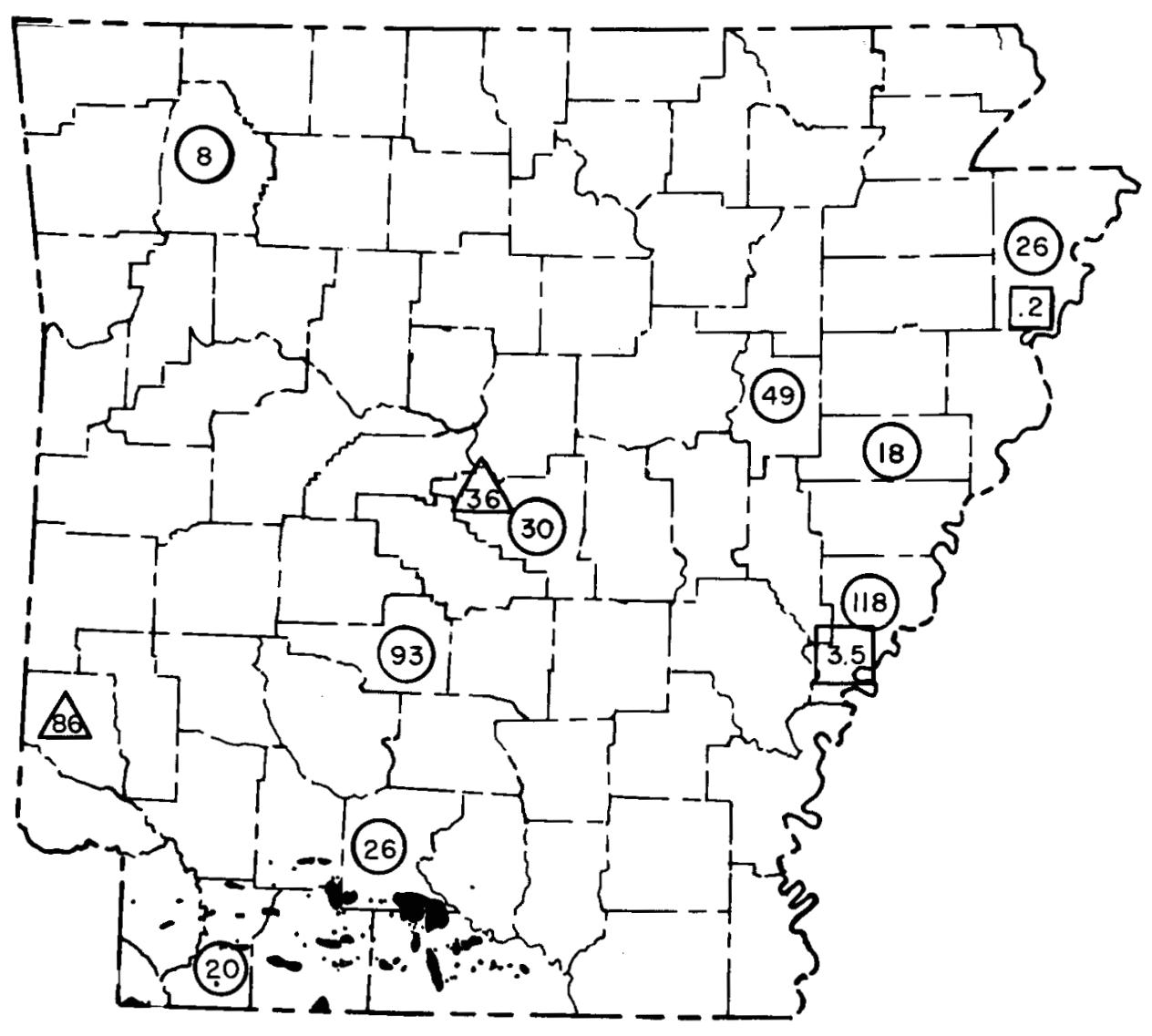

\section{LEGEND}

Flue gas $\mathrm{CO}_{2}$ in MMcfd, gas el ectric plants

$\angle$ Flue gas $\mathrm{CO}_{3}$ in $\mathrm{MMcfd}$, coal electric plants

$\triangle$ Flue gas $\mathrm{CO}_{3}$ in MMcfd, cement plants

$\square$ Liquid $\mathrm{CO}_{3}$ in $\mathrm{MMcfd}$, chemical plants

Oilfields

FIGURE 1. - Oilfields and carbon dioxide sources in Arkansas. 


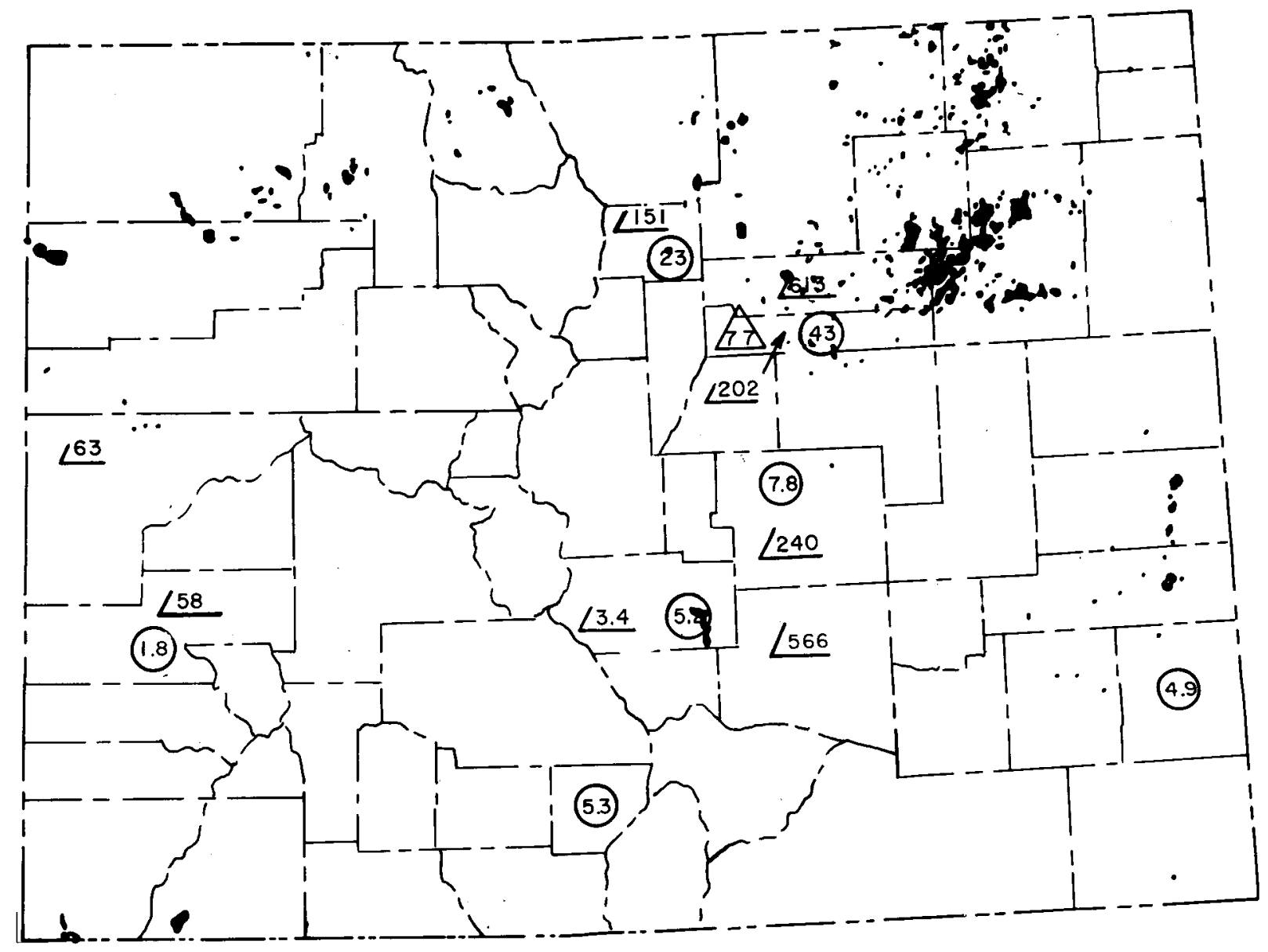

\section{LEGEND}

Flue gas $\mathrm{CO}_{3}$ in MMcfd, gas electric plants

$\angle$ Flue gas $\mathrm{CO}_{2}$ in MMcfd, coal el ectric plants

$\triangle$ Flue gas $\mathrm{CO}_{3}$ in MMcfd, cement plants

$\square$ Liquid $\mathrm{CO}_{3}$ in MMcfd, chemical plants

- Oilfields

FIGURE 2. - Oilfields and carbon dioxide sources in Colorado. 


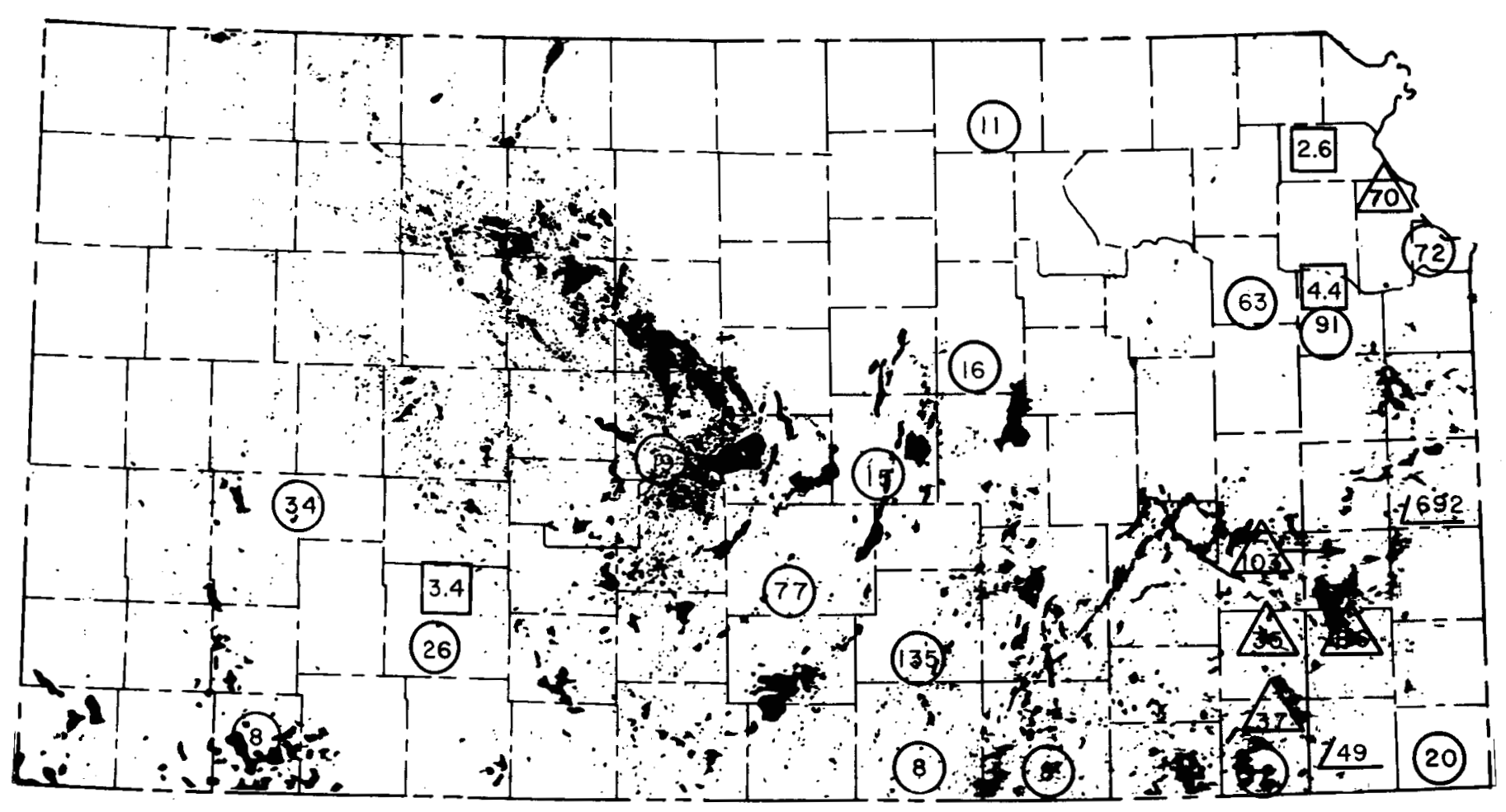

\section{LEGEND}

- Flue gas $\mathrm{CO}_{3}$ in MMcfd, gas el ectric plants

$\angle$ Flue gas $\mathrm{CO}_{2}$ in MMcfd, coal electric plants

$\triangle$ Flue gas $\mathrm{CO}_{3}$ in $\mathrm{MMcfd}$, cement plants

$\square$ Liquid $\mathrm{CO}_{3}$ in $\mathrm{MMcfd}$, chemical plants

- Oilfields

FIGURE 3. - Oilfields and carbon dioxide sources in Kansas. 


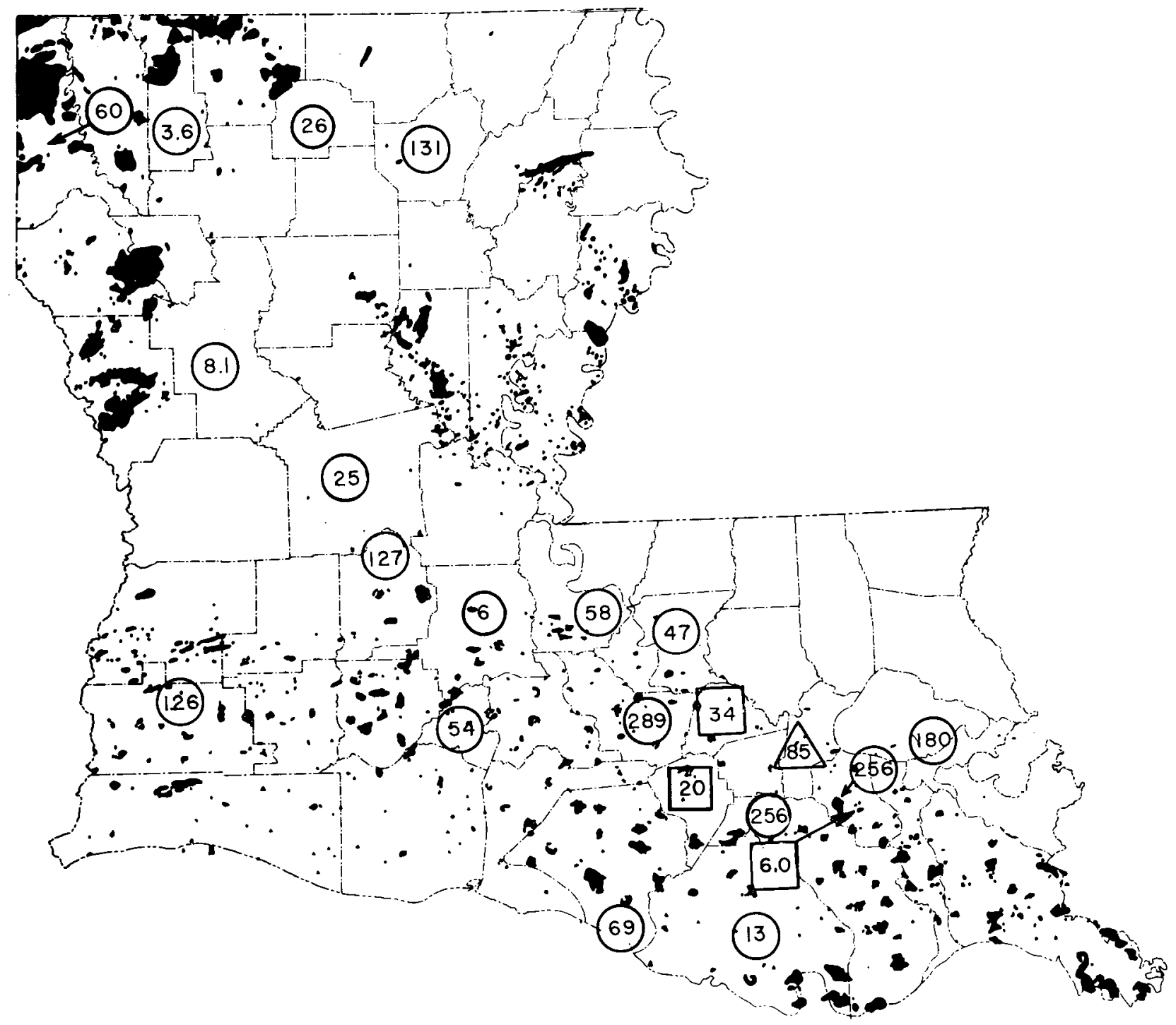

LEGEND

- Flue gas $\mathrm{CO}_{2}$ in MMcfd, gas el ectric plants

$\angle$ Flue gas $\mathrm{CO}_{3}$ in $\mathrm{MMcfd}$, coal electric plants

$\triangle$ Flue gas $\mathrm{CO}_{3}$ in MMcfd, cement plants

$\square$ Liquid $\mathrm{CO}_{2}$ in $\mathrm{MMcfd}$, chemical plants

- Oilfields

FIGURE 4. - Oilfields and carbon dioxide sources in Lovisiana. 


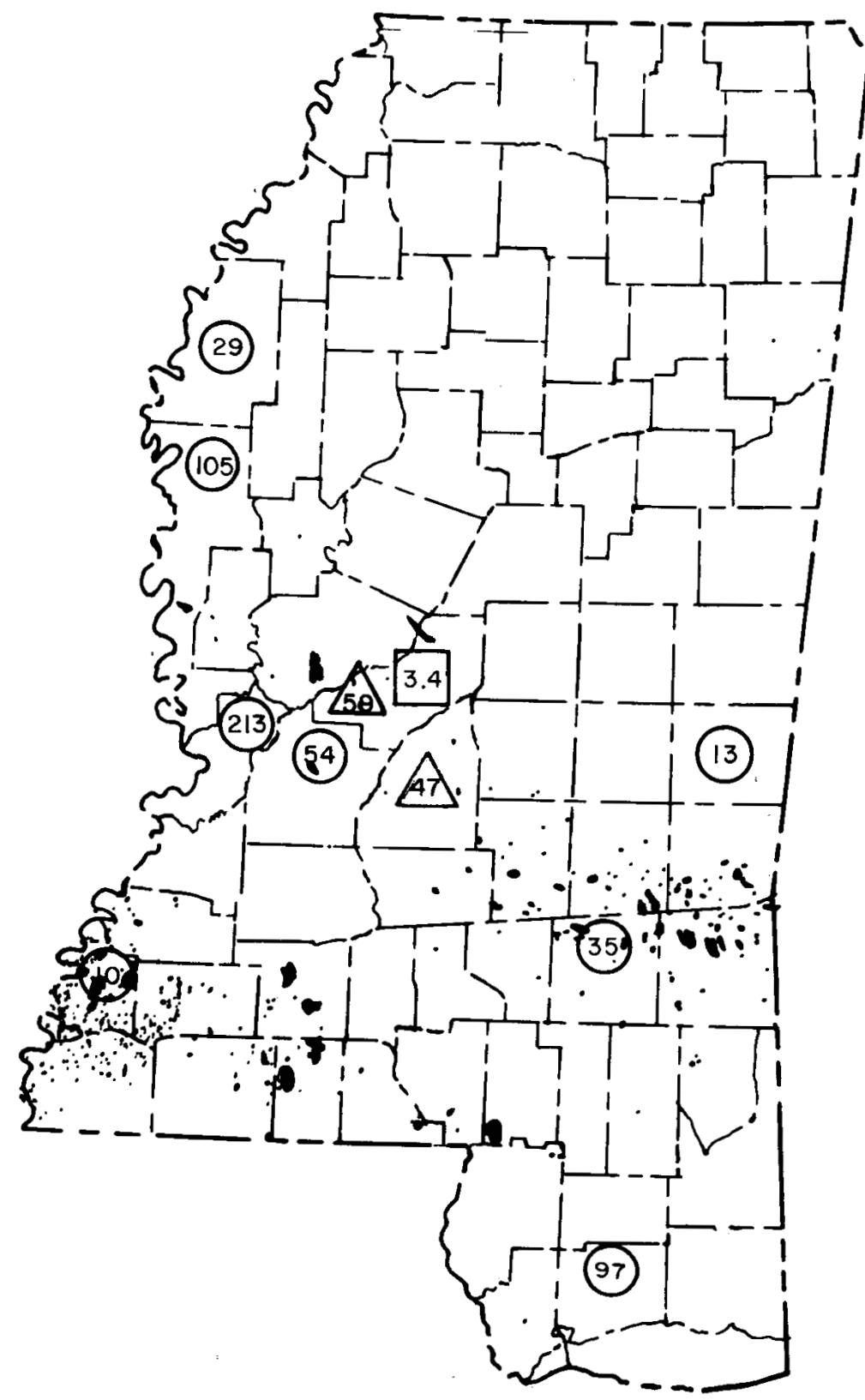

\section{LEGEND}

Flue gas $\mathrm{CO}_{2}$ in MMcfd, gas electric plants

$\angle$ Flue gas $\mathrm{CO}_{3}$ in $\mathrm{MMcfd}$, coal electric plants

$\triangle$ Flue gas $\mathrm{CO}_{3}$ in MMcfd, cement plants

$\square$ Liquid $\mathrm{CO}_{3}$ in $\mathrm{MMcfd}$, chemical plants
Oilfields

FIGURE 5. - Oilfields and carbon dioxide sources in Mississippi. 


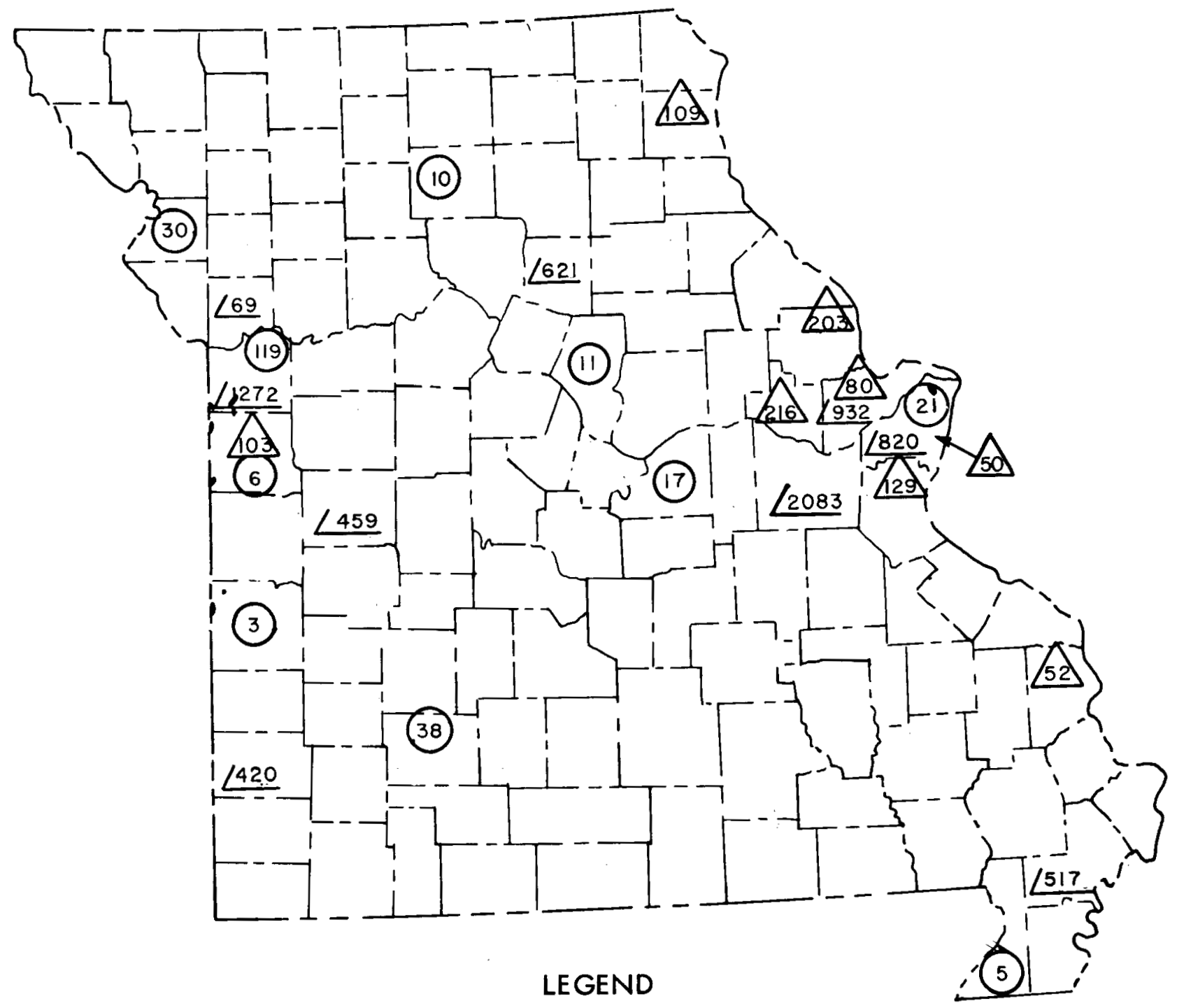

Flue gas $\mathrm{CO}_{2}$ in MMcfd, gas el ectric plants

$\angle$ Flue gas $\mathrm{CO}_{2}$ in MMcfd, coal electric plants

$\triangle$ Flue gas $\mathrm{CO}_{2}$ in MMcfd, cement plants

$\square$ Liquid $\mathrm{CO}_{2}$ in MMcfd, chemical plants

- Oilfields

FIGURE 6. - Oilfields and carbon dioxide sources in Missouri. 


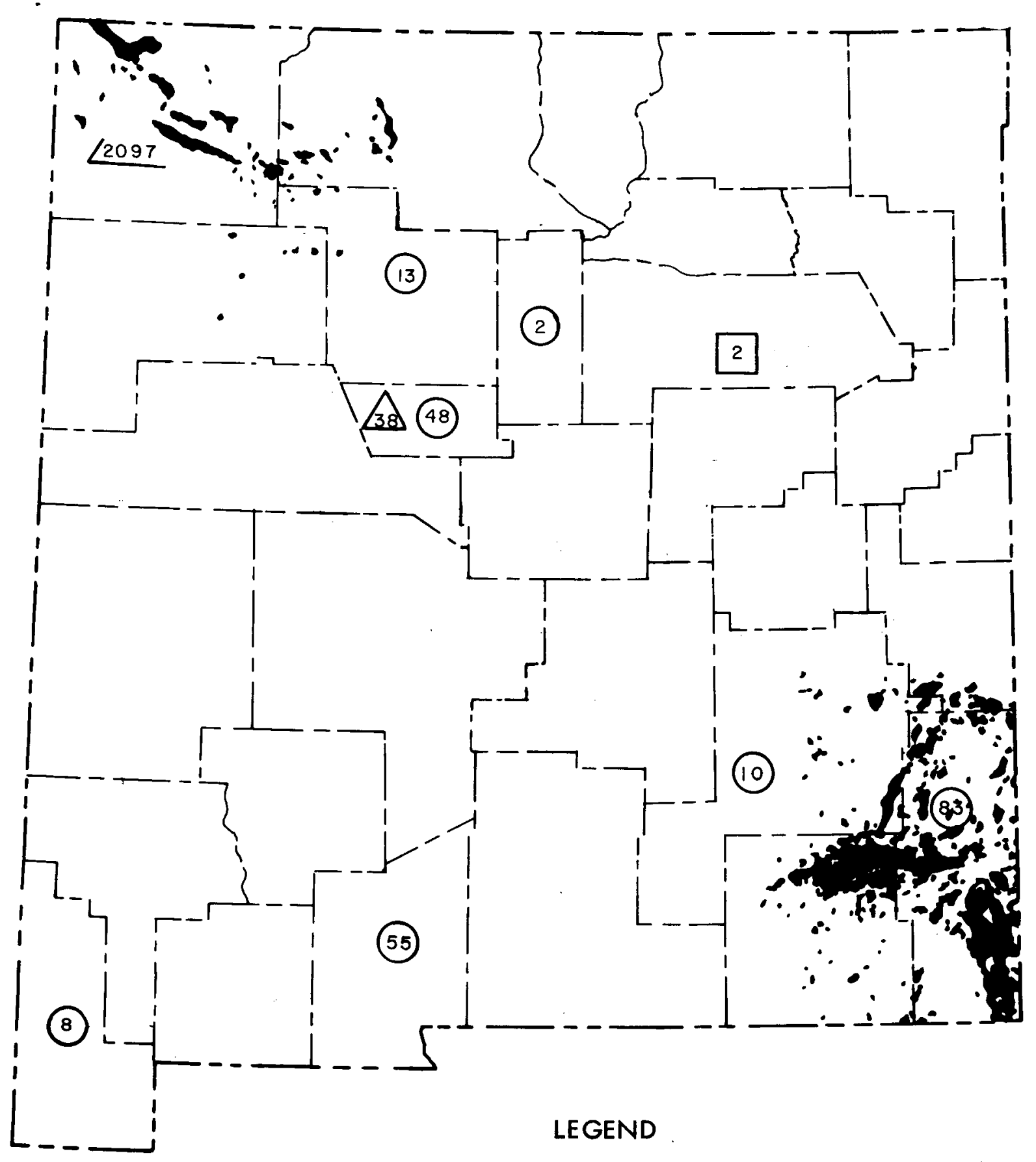

Flue gas $\mathrm{CO}_{3}$ in MMcfd, gas electric plants

$\angle$ Flue gas $\mathrm{CO}_{3}$ in MMcfd, coal electric plants

$\triangle$ Flue gas $\mathrm{CO}_{3}$ in $\mathrm{MMcfd}$, cement plants

L Liquid $\mathrm{CO}_{3}$ in MMcfd, chemical plants

FIGURE 7. - Oilfields and carbon dioxide sources in New Mexico. 


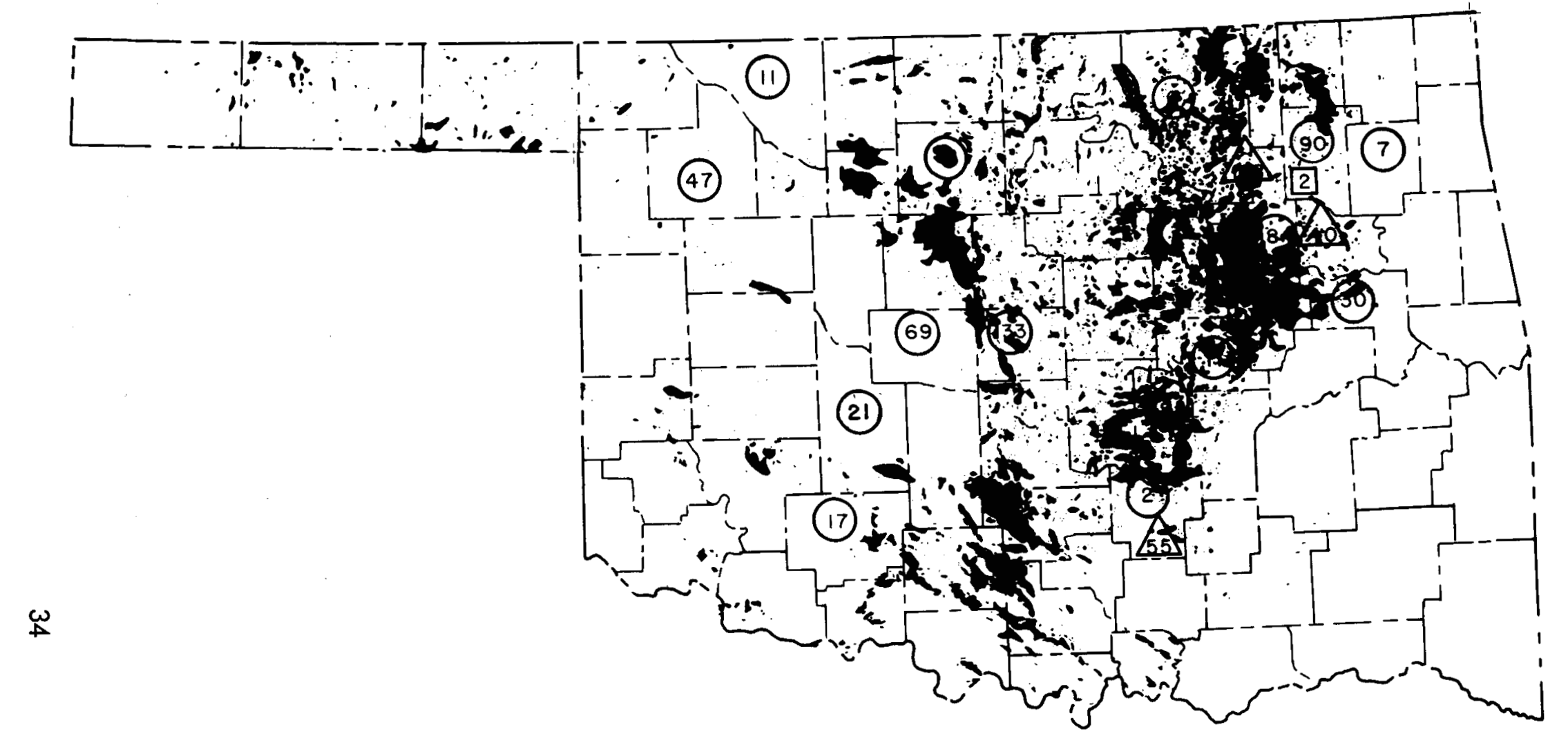

\section{LEGEND}

Flue gas $\mathrm{CO}_{3}$ in MMcfd, gas electric plants

$\angle$ Flue gas $\mathrm{CO}_{2}$ in MMcfd, coal electric plants

$\triangle$ Flue gas $\mathrm{CO}_{3}$ in MMcfd, cement plants

Liquid $\mathrm{CO}_{3}$ in MMcfd, chemical plants

Oilfields

FIGURE 8. - Oilfields and carbon dioxide sources in Oklahoma. 


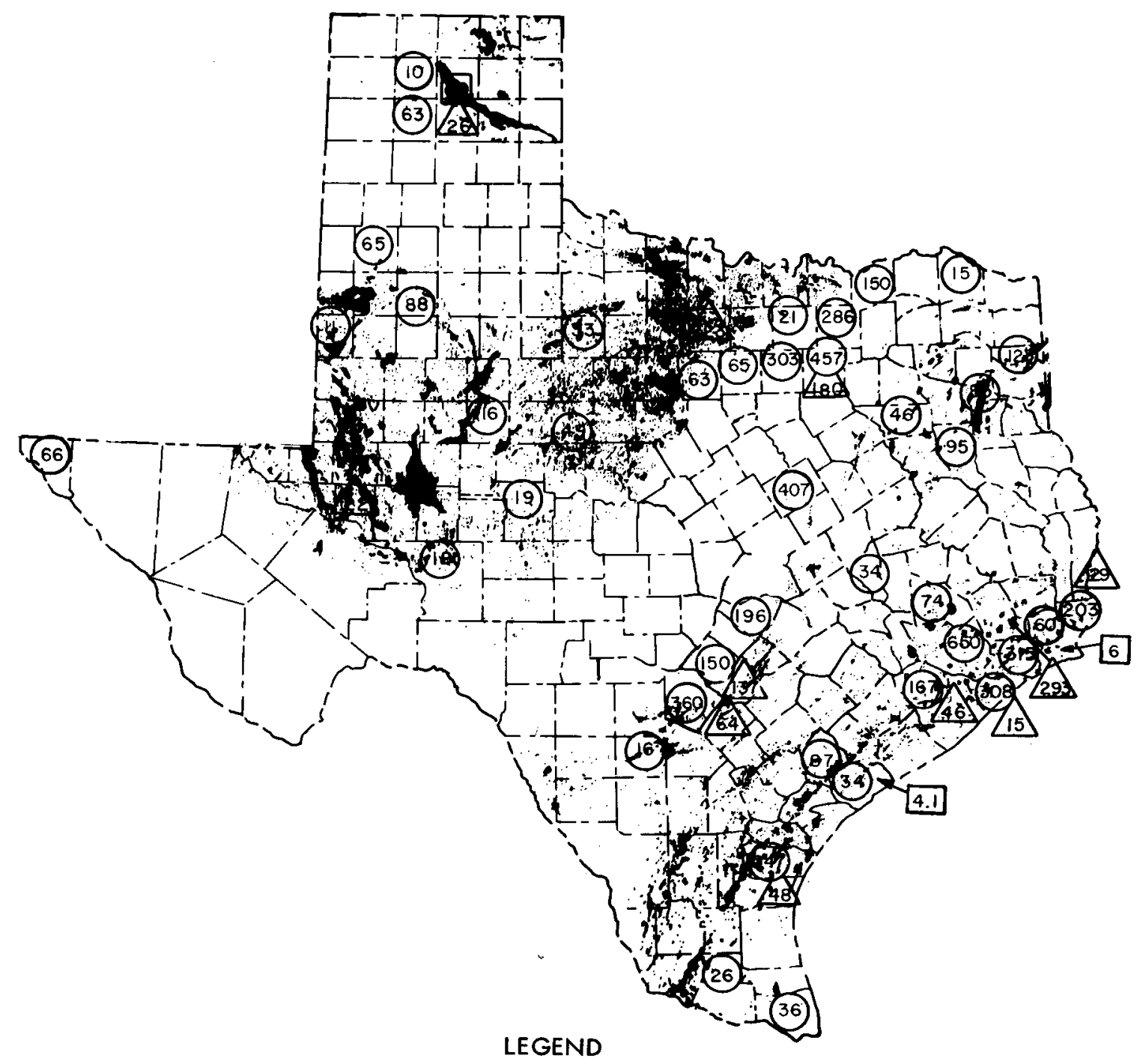

Flue gas $\mathrm{CO}_{2}$ in MMcfd, gas electric plants

$\angle$ Flue gas $\mathrm{CO}_{3}$ in MMcfd, coal el ectric plants

$\triangle$ Flue gas $\mathrm{CO}_{2}$ in MMcfd, cement plants

Liquid $\mathrm{CO}_{z}$ in MMcfd, chemical plants

- Oilfields

FIGURE 9. - Oilfields and carbon dioxide sources in Texas. 